\title{
Anxiety-like behavior and whole-body cortisol responses to components of energy drinks in zebrafish (Danio rerio)
}

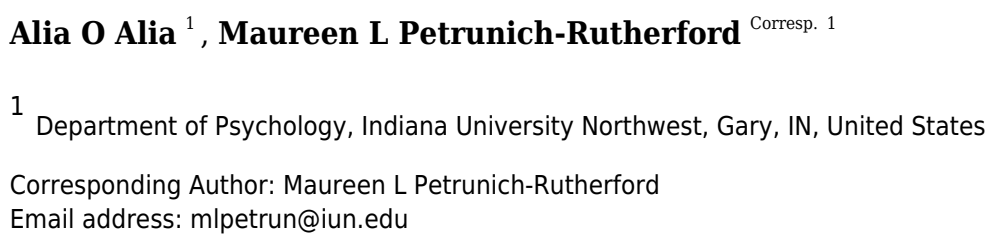

The current study investigated the independent and combined effects of caffeine and taurine on anxiety-like behavior and neuroendocrine responses in adult zebrafish (Danio rerio). Caffeine (1,3,7-trimethylpurine-2,6-dione), the world's most commonly used psychoactive drug, acts as an adenosine receptor blocker and a mild central nervous system stimulant. However, excessive use of caffeine is associated with heightened anxiety levels. Taurine (2-aminoethanesulfonic acid), a semi-essential amino acid synthesized within the human brain, has been hypothesized to play a role in regulating anxiolytic behavior. Caffeine and taurine are two common additives in energy drinks and are often found in high concentrations in these beverages. However, few studies have investigated the interaction of these two chemicals with regards to anxiety measures. A suitable vertebrate to examine anxiety-like behavior and physiological stress responses is the zebrafish, which has shown promise due to substantial physiological and genetic homology with humans. Anxiety-like behavior in zebrafish can be determined by analyzing habituation to novelty when fish are placed into a novel tank and scototaxis (light avoidance) behavior in the light-dark test. Stress-related neuroendocrine responses can be measured in zebrafish by analyzing whole-body cortisol levels. The goal of this study was to determine if exposure to caffeine, taurine, or a combination of the two compounds altered anxiety-like behavior and whole-body cortisol levels in zebrafish relative to control. Zebrafish were individually exposed to either caffeine (100 mg/L), taurine (400 mg/ L), or both for fifteen minutes. Zebrafish in the control group were handled in the same manner but were only exposed to system tank water. After treatment, fish were transferred to the novel tank test or the light-dark test. Behavior was tracked for the first six minutes in the novel tank and fifteen minutes in the light-tark test. Fifteen minutes after introduction to the behavioral task, fish were euthanized for the analysis of whole-body cortisol levels. The results demonstrate that caffeine treatment decreased the amount of exploration in the top of the novel tank and increased scototaxis behavior in the light-dark test, which 
not alter basal levels of anxiety-like behavioral responses nor ameliorated the anxiogenic effects of caffeine on behavior when the two compounds were administered concurrently. None of the drug treatments altered basal levels of whole-body cortisol. The current results of this study suggest that, at least at this dose and time of exposure, taurine does not mitigate the anxiety-producing effects of caffeine when administered in combination, such as with energy drink consumption. 


\section{Anxiety-like behavior and whole-body cortisol responses to components of}

\section{2 energy drinks in zebrafish (Danio rerio)}

3

$4 \quad$ Alia O. Alia ${ }^{1}$ and Maureen L. Petrunich-Rutherford ${ }^{1}$

5

$6 \quad{ }^{1}$ Department of Psychology, Indiana University Northwest, Gary, Indiana, USA

7

8 Corresponding Author:

9 Maureen L. Petrunich-Rutherford

10 Email address: mlpetrun@iun.edu

11

12

13

14

15

16

17

18

19

20

21

22

23

24

25

26

27

28

29

30

31

32

33

34

35 
36

37

38

39

40

41

42

43

44

45

46

47

\begin{abstract}
\end{abstract}
The current study investigated the independent and combined effects of caffeine and taurine on anxiety-like behavior and neuroendocrine responses in adult zebrafish (Danio rerio). Caffeine (1,3,7-trimethylpurine-2,6-dione), the world's most commonly used psychoactive drug, acts as an adenosine receptor blocker and a mild central nervous system stimulant. However, excessive use of caffeine is associated with heightened anxiety levels. Taurine (2aminoethanesulfonic acid), a semi-essential amino acid synthesized within the human brain, has been hypothesized to play a role in regulating anxiolytic behavior. Caffeine and taurine are two common additives in energy drinks and are often found in high concentrations in these beverages. However, few studies have investigated the interaction of these two chemicals with regards to anxiety measures. A suitable vertebrate to examine anxiety-like behavior and physiological stress responses is the zebrafish, which has shown promise due to substantial physiological and genetic homology with humans. Anxiety-like behavior in zebrafish can be determined by analyzing habituation to novelty when fish are placed into a novel tank and scototaxis (light avoidance) behavior in the light-dark test. Stress-related neuroendocrine responses can be measured in zebrafish by analyzing whole-body cortisol levels. The goal of this study was to determine if exposure to caffeine, taurine, or a combination of the two compounds altered anxiety-like behavior and whole-body cortisol levels in zebrafish relative to control.

1 Zebrafish were individually exposed to either caffeine $(100 \mathrm{mg} / \mathrm{L})$, taurine $(400 \mathrm{mg} / \mathrm{L})$, or both for fifteen minutes. Zebrafish in the control group were handled in the same manner but were 
63 only exposed to system tank water. After treatment, fish were transferred to the novel tank test or

64 the light-dark test. Behavior was tracked for the first six minutes in the novel tank and fifteen

65 minutes in the light-tark test. Fifteen minutes after introduction to the behavioral task, fish were

66 euthanized for the analysis of whole-body cortisol levels. The results demonstrate that caffeine

67 treatment decreased the amount of exploration in the top of the novel tank and increased

68 scototaxis behavior in the light-dark test, which supports the established anxiogenic effect of

69 acute exposure to caffeine. Taurine alone did not alter basal levels of anxiety-like behavioral

70 responses nor ameliorated the anxiogenic effects of caffeine on behavior when the two

71 compounds were administered concurrently. None of the drug treatments altered basal levels of

72 whole-body cortisol. The current results of this study suggest that, at least at this dose and time

73 of exposure, taurine does not mitigate the anxiety-producing effects of caffeine when

74 administered in combination, such as with energy drink consumption.

75

76

\section{Introduction}

Caffeine (1,3,7-trimethylpurine-2,6-dione), the world's most widely consumed psychoactive drug, has stimulant-like effects on the central nervous system and overall behavior (Evans \& Battisti, 2018). Widespread use of caffeine is likely due to the positive effects it has on increasing mental alertness and physical endurance as well as reducing fatigue and overall tiredness (Heckman, Weil, \& Gonzalez de Mejia, 2010). However, caffeinated beverages may also be associated with increasing anxiety and other negative health outcomes (Richards \& Smith, 2016), particularly in youth (De Sanctis et al., 2017) and with individuals with certain genetic variants associated with caffeine pharmacokinetics and pharmacodynamics (Nehlig, 2018). The consumption of energy drinks has increased significantly over the last decade in all 
86 age groups surveyed in a recent study (Vercammen, Koma, \& Bleich, 2019). It is likely that

87 energy drinks are popular due to the stimulant-like effects produced by caffeine; thus, energy

88 drinks are commonly consumed by populations such as young adults in college settings for

89 supporting academic demands (Trunzo et al., 2014) and by military service members (Attipoe,

90 Delahanty, Stephens, \& Deuster, 2018). However, as with beverages containing caffeine,

91 excessive consumption of energy drinks is associated with negative health outcomes like adverse

92 cardiac events (for review, see Higgins, Babu, Deuster, \& Shearer, 2018). Adolescents are more

93 at risk to experiencing ill effects of energy drink consumption (Curran \& Marczinski, 2017). In

94 addition, energy drinks are frequently consumed with alcohol, a practice which is associated with

95 increased risky decision making (Manchester, Eshel, \& Marion, 2017) and increased risk for

96 negative health consequences in young adults (Caviness, Anderson, \& Stein, 2017).

Although caffeine is one of the primary chemicals present in energy drinks, another

98 additive found in high concentrations is taurine. Taurine (2-aminoethanesulfonic acid) is

99 considered a semi-essential amino acid, as is it not used in protein synthesis (Ripps \& Shen,

100 2012). However, taurine does play other critical roles, particularly in the central nervous system,

101 such as by helping to regulate cell volume and modulate neurotransmission in the brain (Oja \&

102 Saransaari, 2017). In addition, taurine has been hypothesized to play a role in anxiolytic

103 behavior in rodents (Chen et al., 2004; El Idrissi et al., 2009; Francisco \& Guedes, 2015; Kong et

104 al., 2006; McCool \& Chappell, 2007; Wu et al., 2017; Zhang \& Kim, 2007). The effects of

105 caffeine and taurine in combination have mainly been studied in the context of physical

106 performance and cardiovascular function. Administration of caffeine and taurine in combination

107 altered measures of cardiovascular function (Bichler, Swenson, \& Harris, 2006) and elevated

108 mental performance and mood (Seidl, Peyrl, Nicham, \& Hauser, 2000) over placebo in human 
109 participants. When these two components were studied alone and in combination, taurine 110 counteracted the effects of caffeine on cardiovascular function (Schaffer et al., 2014), mitigated

111 some of the effects of caffeine on cognitive measures (Giles et al., 2012), reduced caffeine-

112 induced physiological alterations associated with cycling performance (Warnock, Jeffries,

113 Patterson, \& Waldron, 2017), and attenuated the effects of caffeine on specific parameters of

114 reaction time (Peacock, Martin, \& Carr, 2013) in human subjects. However, in vitro, taurine did

115 not alter caffeine-induced effects in human cardiac muscle tissue (Chaban et al., 2017) or mouse

116 skeletal muscle tissue (Tallis, Higgins, Cox, Duncan, \& James, 2014). In other measures in a

117 variety of animal models, caffeine and taurine have synergistic effects, such as on sleep

118 parameters in Drosophila (Lin et al., 2010), plasma calcium levels in rats (Owoyele, Oyewole,

119 Biliaminu, \& Alashi, 2015), locomotor activity in mice (Kimura, Ushijima, Hiraki, Kimura, \&

120 Ono, 2009), and memory and attention in rats (Valle et al., 2018). Thus, the specific impact of

121 caffeine and taurine appears to be dependent on the physiological and behavioral parameters

122 under investigation. Although caffeine and taurine can modulate anxiety-like states on their own,

123 little is known regarding the impact of these two popular energy drink components in

124 combination.

125

The zebrafish (Danio rerio) animal model is rapidly becoming an attractive model organism in neuropharmacology research due to its low cost and ease of maintenance; in 127 addition, the nervous and endocrine systems regulating biological and behavioral responses to 128 stress are highly conserved (Stewart et al., 2012). Stress and anxiety-like states can be inferred in 129 the zebrafish model by measuring various behavioral responses such as shoaling, immobility, 130 erratic movements, and the detection of jumping in response to various stimuli such as the 131 introduction of pharmaceuticals, visual stimuli, and alarm pheromones (Egan et al., 2009; 
132 Maximino et al., 2014; K. Wong et al., 2010). The novel tank test is a well-validated measure of

133 anxiety-like behavior in zebrafish and involves measuring freezing and exploratory behavior

134 upon introduction to a new tank (Kysil et al., 2017; Mezzomo, Silveira, Giuliani, Quadros, \&

135 Rosemberg, 2016; Raymond et al., 2012). Another behavioral paradigm, the light-dark test,

136 measures anxiety-like behavior in the form of scototaxis, or light avoidance, in response to

137 pharmacological or behavioral manipulation (Maximino, Marques de Brito, Dias, Gouveia, \&

138 Morato, 2010; Stewart et al., 2011). Stress responses can also be assessed through measuring

139 neuroendocrine responses, namely cortisol, elicited by specific stimuli (Cachat et al., 2010;

140 Canavello et al., 2011). The hypothalamic-pituitary-interrenal (HPI) axis of teleost species, such

141 as zebrafish, is homologous to the hypothalamic-pituitary adrenal (HPA) axis of mammals

142 (Nesan \& Vijayan, 2013; Wendelaar Bonga, 1997). Thus, exposure to pharmacological

143 compounds will elicit behavioral and neuroendocrine effects in the fish that may generally model

144 the effects these compounds have in humans.

145 Consistent with findings in rodent models and human subjects, zebrafish exposed to

146 caffeine at a variety of ages demonstrate anxiety-like behavior (Egan et al., 2009; Richendrfer,

147 Pelkowski, Colwill, \& Creton, 2012; Rosa et al., 2018; Schnörr, Steenbergen, Richardson, \&

148 Champagne, 2012; Steenbergen et al., 2011; K. Wong et al., 2010). The specific anxiogenic

149 effect of caffeine is possibly due to antagonism at $\mathrm{A}_{1}$ adenosine receptors (Maximino, Lima,

150 Olivera, Picanço-Diniz, \& Herculano, 2011). Acute exposure to taurine, on the other hand, is

151 associated with anxiolytic effects on behavior (Mezzomo, Silveira, Giuliani, Quadros, \&

152 Rosemberg, 2016). The modulation of anxiety-like behavior by taurine in zebrafish may be

153 induced by blunting neuroendocrine cortisol responses to stress (as observed in Mezzomo et al.,

154 2019). Similarly, glucocorticoid hormone regulation has been proposed as a possible mechanism 
155 by which taurine modulates anxiety-like behavior in unpredictable chronic stress exposure in

156 rodents (Wu et al., 2017). However, whether taurine mitigates the anxiogenic effects of caffeine

157 via HPA/HPI regulation is currently not known.

158 Thus, in this study, the effects of caffeine and taurine on anxiety-like behavior and

159 neuroendocrine responses were explored. The purpose of this study was to determine if acute

160 exposure to caffeine, taurine, or both altered anxiety-like behavior and whole-body cortisol

161 levels in zebrafish. If caffeine operates as an anxiogenic in zebrafish as expected, then the fish

162 will exhibit more anxiety-like behavior and display increased whole-body cortisol levels relative

163 to control. If taurine operates as an anxiolytic in zebrafish, then the fish exposed to taurine

164 should have decreased cortisol levels and exhibit decreased anxiety-like behavior, such as

165 spending more time in the upper portion of the tank during the novel tank test or entering the

166 light zone more frequently in the light-dark test. If taurine modulates the effects of caffeine,

167 taurine should mitigate any caffeine-induced anxiety-like effects on behavior and increases in

168 whole-body cortisol when the fish are acutely exposed to both drugs simultaneously. This study

169 will potentially aid in elucidating the effects of caffeine and taurine when co-administered

170 acutely. In addition, the findings from this study will provide insight on the interaction between

171 chemicals commonly found in energy drinks and whether the modulation of anxiety-like

172 behavior is related to the activity of the neuroendocrine stress axis.

173

Methods and Materials

175

176

177

Animals and Housing

Wild-type, adult zebrafish $(\mathrm{N}=139)$ were purchased from Carolina Biological Supply

(Burlington, NC). Upon arrival to the facility, the fish were maintained on a circulating system 
178 on a 14:10 light:dark cycle at a density of approximately 5-6 fish per liter. Fish were fed flake

179 food once per day and dried brine shrimp once per day. The internal environment of the housing

180 tanks was maintained at a temperature of $26 \pm 2^{\circ} \mathrm{C}$. Animals were housed and maintained in

181 accordance with ethical guidelines (Harper \& Lawrence, 2016; National Research Council, 2011;

182 Westerfield, 2000). All fish were allowed to acclimate to the facility for at least a week before

183 any experimental procedures were conducted (Dhanasiri, Fernandes, \& Kiron, 2013). All

184 experimental procedures involving animals were performed between 9:00 a.m. and 1:00 p.m.

185

186

187

188

189

190

191

192

193

194

195

196

197

198 199 for behavioral analysis.

200 Novel Tank Test (Experiment 1)

\section{Drug Administration}

Drugs were purchased from Santa Cruz Biotechnology (Dallas, TX). On the day of the experiment, housing tanks were removed from the system and placed in the experimentation room thirty minutes prior to treatment to allow for habituation. Individual fish were selected at random, carefully netted from the tank, and placed in a tank containing $1 \mathrm{~L}$ of either a drug or control solution for a duration of 15 minutes. There were four independent conditions: control (system water), caffeine (1,3,7-trimethylpurine-2,6-dione), taurine (2-aminoethanesulfonic acid), or a combination of caffeine/taurine. Fish were either immersed in a solution of either caffeine $(\mathrm{N}=29)$ at $100 \mathrm{mg} / \mathrm{L}$ (Maximino et al., 2014), taurine $(\mathrm{N}=30)$ at $400 \mathrm{mg} / \mathrm{L}$ (Mezzomo et al., 2016), or caffeine and taurine combined $(\mathrm{N}=29)$ at $100 \mathrm{mg} / \mathrm{L}$ and $400 \mathrm{mg} / \mathrm{L}$, respectively.

Subjects in the control group $(\mathrm{N}=51)$ were simply immersed in system water for 15 minutes. Treatment solutions were replaced after every subject. Immediately following treatment, each subject was transferred to either a novel tank (Experiment 1) or light-dark tank (Experiment 2)

Peer] reviewing PDF | (2019:04:36362:2:0:NEW 18 Jul 2019) 
After drug treatment, individual fish $(\mathrm{N}=100$ total) were placed in a trapezoidal tank 202 (15.2 cm height $\times 27.9 \mathrm{~cm}$ top $\times 22.5 \mathrm{~cm}$ bottom $\times 7.1 \mathrm{~cm}$ width). The tank was positioned to 203 allow for recording of behavior from the wide side of the tank, using a camera placed on a tripod. 204 The first six minutes of behavior was recorded and subsequently analyzed by Ethovision XT 205 software (Noldus, Leesburg, VA), which was generously provided as part of the Faculty for 206 Undergraduate Neuroscience (FUN) Equipment Loan Program. Behavioral measures included 207 the total distance traveled $(\mathrm{cm})$, mean speed during ambulation $(\mathrm{cm} / \mathrm{s})$, immobility duration $(\mathrm{s})$, 208 number of times fish were immobile, latency to first top entry (s), total time in top (s), distance in 209 top $(\mathrm{cm})$, and number of entries to top. The percentage of fish from each group that did not re210 enter the top zone after being introduced to the novel tank $(\mathrm{N}=18$ total) was also calculated.

211 These samples were not included in the analysis of the latency to top measure but were included 212 in all other behavioral measures. One subject was not included in behavioral analyses due to 213 corruption of the video file.

Light-Dark Test (Experiment 2)

After the drug treatment, individual fish $(\mathrm{N}=39$ total $)$ were placed in a rectangular tank 217 (approximately $15 \mathrm{~cm} \times 30 \mathrm{~cm} \times 20 \mathrm{~cm}$ ) with a water depth of $3 \mathrm{~cm}$. The dark side of the tank 218 (sides and bottom) was covered with black plastic aquarium background and the other side was 219 left uncovered (Magno, Fontes, Gonçalves, \& Gouveia, 2015; Maximino et al., 2010). The 220 behavior of the fish was recorded from above the tank for fifteen minutes with a Logitech C922x 221 Pro Stream Webcam. Video files were uploaded to and analyzed with BehaviorCloud motion222 tracking software (https://www.behaviorcloud.com/, San Diego, CA). Six behavioral measures 223 were quantified for each fish: total distance traveled $(\mathrm{cm})$, mean speed during ambulation $(\mathrm{cm} / \mathrm{s})$, 
224 immobility duration (s), number of entries to the light zone, total time spent in light zone (min),

225 and total distance traveled in the light zone $(\mathrm{cm})$.

226 Euthanasia

227 Fifteen minutes after each subject was introduced to the respective behavioral task, the

228 fish were netted from the tank and placed into $30 \mathrm{ml}$ euthanasia solution $(0.1 \%$ clove oil in

229 system water) for approximately 60 seconds (Davis et al., 2015; D. Wong, von Keyserlingk,

230 Richards, \& Weary, 2014). Once the subjects displayed no movement or responsiveness, the

231 bodies were gently dried, placed in a microcentrifuge tube, and were stored at $-20^{\circ} \mathrm{C}$ until the

232 cortisol extraction was performed.

233 Cortisol Extraction/Assay

234 Cortisol extraction and analysis procedures were adapted from previously published

235 methods (Cachat et al., 2010; Canavello et al., 2011). Briefly, whole-body samples were thawed

236 and individually weighed. Samples were cut into smaller pieces with a scalpel and placed in $1 \mathrm{ml}$

237 of $25 \mathrm{mM}$ of ice-cold phosphate buffer solution (PBS). Each sample was homogenized for 30-60

238 seconds and placed back on ice. Diethyl ether $(5 \mathrm{ml})$ was added to each sample and thoroughly

239 vortexed, then centrifuged at $2500 \mathrm{rpm}$ for 15 minutes. Following the centrifugation, the organic

240 layer containing the cortisol was removed from the sample and placed in a separate tube. The

241 addition of ether, vortexing, centrifugation, and organic layer removal was repeated two more

242 times to maximize the amount of cortisol extracted from each sample. The samples from

243 Experiment 1 were allowed to dry at room temperature under a fume hood until the ether layer

244 was fully evaporated; samples from Experiment 2 were dried with a light stream of air. In both

245 procedures, samples were dried until a yellow oil containing cortisol remained.

Peer] reviewing PDF | (2019:04:36362:2:0:NEW 18 Jul 2019) 
After the samples were dry, $1 \mathrm{ml}$ of ice-cold PBS was added to each tube, and a

247

248

249

250

251

252

253

254

255

256

257

258

259

260

261

262

263

264

265

266

267 268

commercially-available enzyme-linked immunosorbent assay (ELISA) kit (Salimetrics,

Carlsbad, CA) was used to assess cortisol levels. ELISA procedures were conducted according to manufacturer instructions. Binding values for each sample was compared to a standard curve generated by My Curve Fit software (https://mycurvefit.com/). Cortisol levels were normalized to body weights of each sample and are displayed in ng cortisol/g body weight. Four samples from Experiment 1 were excluded from the cortisol analysis due to methodological errors incurred during the extraction procedure.

\section{Data Analysis}

Behavioral and cortisol dependent measures were expressed as the mean \pm standard error of the mean and were analyzed using a two-way analysis of variance (ANOVA) with caffeine (levels: yes, no) and taurine (levels: yes, no) as the independent variables. Group means were compared by a Tukey post-hoc test when appropriate. The percentage of each group that did not explore the top zone of the novel tank test was analyzed with a chi-squared test. All analyses were conducted using JASP software (https://jasp-stats.org/). Results were considered statistically significant if $\mathrm{p}<0.05$.

\footnotetext{
Results

Anxiety-like responses of subjects were determined by assessing behavioral measures exhibited within the novel tank test and light-dark test. In addition, whether neurochemical measures of anxiety were altered by drug treatment was assessed by measuring whole-body cortisol levels after each of the behavioral tasks. Measures of each behavioral test were broken down into three discrete domains: motor activity, immobility, and exploration. In the novel tank
} 
269 test (Experiment 1), motor activity was assessed by examining the total distance moved overall

270 and the mean speed of the subjects (while mobile) within the tank. The second domain assessed

271 the number of times the subjects were immobile and the total duration of immobility (s). The

272 third domain included the activity in the top zone of the novel tank; this included assessing the

273 distance moved in the top zone $(\mathrm{cm})$, the number of times the subjects entered the top zone, the

274 time spent in the top zone (s), and the latency to first top entry (s). The percentage of subjects in 275 each group that did not explore the top zone was also calculated. In the light-dark test

276 (Experiment 2), motor activity was represented by the total distance moved overall and the mean 277 speed of the subjects (while mobile) within the tank. The second domain assessed freezing by 278 measuring the duration of immobility (s). The third domain included the activity in the light 279 zone of the tank; this included assessing the number of times the subjects entered the light zone, 280 the distance moved in the light zone $(\mathrm{cm})$, the time spent in the light zone (s), the number of 281 crossings from one compartment to the other, and the latency to re-enter the light zone after the 282 first visit to the dark zone (s).

283 Experiment 1

$284 \quad$ Motor activity in the novel tank test. The total distance traveled and the mean ambulatory 285 speed in the novel tank test is illustrated in Figure 1. A two-factor ANOVA revealed no 286 significant main effect of caffeine $(F(1,95)=2.898, p=0.092)$, no significant main effect of 287 taurine $(\mathrm{F}(1,95)=1.101, \mathrm{p}=0.297)$, and no significant interaction between caffeine and taurine $288(\mathrm{~F}(1,95)=2.263, \mathrm{p}=0.136)$ on the total distance traveled in the novel tank test (Figure 1A). A 289 two-factor ANOVA indicated a marginally significant main effect of caffeine $(F(1,95)=3.214, p$ $290=0.076)$, no significant main effect of taurine $(F(1,95)=0.061, p=0.806)$, but no significant 291 interaction between caffeine and taurine $(\mathrm{F}(1,95)=0.047, \mathrm{p}=0.829)$ on the mean ambulatory 
292 speed traveled in the novel tank test (Figure 1B). Thus, it appears that caffeine and taurine, either 293 alone or in combination, did not significantly affect general motor activity of adult zebrafish in 294 the novel tank test.

295 Freezing behavior in the novel tank test. Freezing behavior (Figure 2) displayed in 296 zebrafish in the novel tank test can be used as an indication of anxiety-like behavior induced by 297 treatment. As the number of freezing bouts or time spent immobile increases, it can be inferred 298 that the subjects are experiencing higher levels of anxiety. A two-factor ANOVA revealed no 299 significant main effect of caffeine $(F(1,95)=1.674, p=0.199)$, no significant main effect of 300 taurine $(F(1,95)=0.534, p=0.467)$, and no significant interaction between caffeine and taurine $301 \quad(F(1,95)=0.339, p=0.562)$ on the number of immobility bouts in the novel tank test (Figure 302 2A). A two-factor ANOVA indicated no significant main effect of caffeine $(F(1,95)=1.004, p=$ 303 $0.319)$, no significant main effect of taurine $(F(1,95)=0.062, \mathrm{p}=0.805)$, and no significant 304 interaction between caffeine and taurine $(F(1,95)=0.184, \mathrm{p}=0.669)$ on the total time spent 305 immobile in the novel tank test (Figure 2B). Thus, it appears that caffeine and taurine, either alone or in combination, did not significantly affect freezing behavior of adult zebrafish in the 307 novel tank test. Exploratory behavior in the novel tank test. Figure 3 displays the mean \pm SEM for each group for each of the four exploratory measures of interest in the novel tank test. If the subjects are less exploratory (e.g., spend less time in the top, enter the top fewer times, etc.), then it can

311 be inferred that the subjects are experiencing more anxiety. A two-factor ANOVA revealed no 312 significant main effect of caffeine $(F(1,95)=2.019, \mathrm{p}=0.159)$, no significant main effect of 313 taurine $(F(1,95)=2.150, p=0.146)$, and no significant interaction between caffeine and taurine $314(\mathrm{~F}(1,95)=2.701, \mathrm{p}=0.104)$ on the distance traveled in the top zone of the novel tank test (Figure 
$3153 \mathrm{~A})$. A two-factor ANOVA indicated a significant main effect of caffeine $(\mathrm{F}(1,95)=6.379, \mathrm{p}=$

3160.013 , caffeine $<$ no caffeine $)$, no significant main effect of taurine $(F(1,95)=0.515, p=0.475)$,

317 but no significant interaction between caffeine and taurine $(F(1,95)=0.021, p=0.886)$ on the

318 number of entries to the top zone of the novel tank test (Figure 3B). A two-factor ANOVA

319 revealed no significant main effect of caffeine $(\mathrm{F}(1,95)=0.361, \mathrm{p}=0.550)$, no significant main

320 effect of taurine $(\mathrm{F}(1,95)=1.480, \mathrm{p}=0.227)$, and no significant interaction between caffeine and

321 taurine $(\mathrm{F}(1,95)=2.372, \mathrm{p}=0.127)$ on the time spent in the top zone of the novel tank test

322 (Figure 3C). A two-factor ANOVA indicated no significant main effect of caffeine $(\mathrm{F}(1,77)=$

$3230.786, p=0.378)$, a significant main effect of taurine $(F(1,77)=4.308, p=0.041$, taurine $<$ no

324 taurine), but no significant interaction between caffeine and taurine $(\mathrm{F}(1,77)=0.567, \mathrm{p}=0.454)$

325 on the latency to enter the top zone of the novel tank test (Figure 3D). It is of note that, for this

326 behavioral task, not all fish returned to the top (see Table 1) and thus could not be included in

327 this analysis. Table 1 displays the percentage of fish from each group that did not explore the top

328 portion of the novel tank. Almost half (42.1\%) of the caffeine-treated group did not explore the

329 top but fewer than $10 \%$ of the fish exposed to the control or taurine conditions did not explore

330 the top. About $25 \%$ of fish exposed to the mixed drug treatment failed to explore the top zone in

331 the novel tank test. These group differences were significant according to a chi-squared test $\left(\chi^{2}\right.$

$332(3, \mathrm{~N}=99)=12.02, \mathrm{p}=0.007)$. Thus, the pattern of data suggests that caffeine treatment

333 decreased the tendency to explore the top, and that fish that did re-enter the top took more time

334 to do so after treatment with caffeine alone.

335 Although some of the exploratory measures did not reach the criterion for statistical

336 significance, in general, caffeine-treated fish appeared to demonstrate less exploratory behavior

337 in the top zone, whereas taurine generally did not alter overall exploration besides shortening the 
338 latency to explore the top zone. The data loosely suggest that when caffeine and taurine were co-

339 administered, taurine may have mitigated some of the effects of caffeine on exploration (e.g.,

340 increased distance and time spent in the top zone and decreased the latency to enter the top zone

341 of the novel tank test); however, a higher dose and/or longer course of exposure to taurine is

342 likely necessary to elicit any significant effect on caffeine-induced anxiety-like behavior in the

343 novel tank test.

Whole-body cortisol levels post-novel tank test. A neurochemical marker of anxiety was

345 determined by analyzing whole-body cortisol levels of each subject (Figure 4). A two-factor

346 ANOVA revealed no significant main effect of caffeine $(F(1,92)=0.189, p=0.665)$, no

347 significant main effect of taurine $(F(1,92)=0.283, p=0.596)$, and no significant interaction

348 between caffeine and taurine $(\mathrm{F}(1,92)=0.660, \mathrm{p}=0.419)$ on whole body cortisol levels (Figure

349 4). Thus, it does not appear that acute exposure to different components of energy drinks altered

350 stress hormone responses, at least when cortisol was assessed fifteen minutes after introduction

351 to the novel tank test.

352 Experiment 2

353 Motor activity in the light-dark test. Similar to the novel tank test, the total distance

354 traveled and the mean ambulatory speed (Figure 5) can be used as markers for general motor

355 activity in the light-dark test. A two-factor ANOVA revealed a significant main effect of caffeine

$356(\mathrm{~F}(1,35)=17.791, \mathrm{p}<0.001$, caffeine $<$ no caffeine $)$, no significant main effect of taurine

$357(F(1,35)=0.336, p=0.566)$, but no significant interaction between caffeine and taurine $(F(1,35)$

$358=0.343, \mathrm{p}=0.562$ ) on the total distance traveled in the light-dark test (Figure 5A). A two-factor

359 ANOVA indicated no significant main effect of caffeine $(F(1,35)=2.074, p=0.159)$, no

360 significant main effect of taurine $(F(1,35)=0.245, p=0.624)$, and no significant interaction 
361 between caffeine and taurine $(F(1,35)=0.013, p=0.911)$ on the mean speed traveled (while

362 ambulatory) in the light-dark test (Figure 5B). Thus, it appears that caffeine, but not taurine,

363 significantly decreased the total distance traveled by adult zebrafish in the light-dark test. None

364 of the treatments altered mean swimming speed of the subjects.

$365 \quad$ Freezing behavior in the light-dark test. Freezing behavior (Figure 6) displayed in

366 zebrafish can be used as an indication of anxiety-like behavior induced by treatment. A two-

367 factor ANOVA indicated a significant main effect of caffeine $(F(1,35)=27.792, p<0.001$,

368 caffeine $>$ no caffeine), no significant main effect of taurine $(F(1,35)=0.764, p=0.388)$, but no

369 significant interaction between caffeine and taurine $(F(1,35)=0.699, p=0.409)$ on the total time

370 spent immobile in the novel tank test (Figure 6). Thus, it appears that caffeine, but not taurine,

371 significantly increased the time spent immobile by adult zebrafish in the light-dark test. This may

372 explain why the total distance traveled was less for caffeine-treated fish (see Figure 5A).

373 Exploratory behavior in the light-dark test. Figure 7 displays the mean \pm SEM for each

374 group for each of the three exploratory measures of interest in the light-dark test. If the subjects

375 are less exploratory (e.g., spend less time in the light zone, enter the light zone fewer times, etc.)

376 in the light-dark test, then it can be inferred that the subjects are experiencing more anxiety. A

377 two-factor ANOVA revealed a significant main effect of caffeine $(F(1,35)=19.033, p<0.001$,

378 caffeine $<$ no caffeine), no significant main effect of taurine $(F(1,35)=0.020, p=0.887)$, but no

379 significant interaction between caffeine and taurine $(F(1,35)=0.261, p=0.613)$ on the distance

380 traveled in the light zone of the light-dark test (Figure 7A). A two-factor ANOVA indicated a

381 significant main effect of caffeine $(\mathrm{F}(1,35)=30.364, \mathrm{p}<0.001$, caffeine $<$ no caffeine $)$, no

382 significant main effect of taurine $(F(1,35)=0.122, \mathrm{p}=0.729)$, but no significant interaction

383 between caffeine and taurine $(F(1,35)=0.639, \mathrm{p}=0.430)$ on the number of entries to the light 
384 zone of the light-dark test (Figure 7B). A two-factor ANOVA indicated a significant main effect 385 of caffeine $(F(1,35)=20.088, p<0.001$, caffeine $<$ no caffeine $)$, no significant main effect of 386 taurine $(\mathrm{F}(1,35)=0.024, \mathrm{p}=0.877)$, but no significant interaction between caffeine and taurine $387(\mathrm{~F}(1,35)=0.137, \mathrm{p}=0.714)$ on the number of crossings in the tank from one compartment to the 388 other in the light-dark test (Figure 7C). A two-factor ANOVA revealed no significant main effect 389 of caffeine $(F(1,35)=1.188, p=0.283)$, no significant main effect of taurine $(F(1,35)=2.107, p$ $390=0.155)$, and no significant interaction between caffeine and taurine $(F(1,35)=2.961, \mathrm{p}=0.094)$ 391 on the time spent in the light zone of the light-dark test (Figure 7D). A two-factor ANOVA 392 revealed no significant main effect of caffeine $(\mathrm{F}(1,35)=2.426, \mathrm{p}=0.128)$, no significant main 393 effect of taurine $(F(1,35)=1.411, p=0.243)$, and no significant interaction between caffeine and 394 taurine $(\mathrm{F}(1,35)=1.320, \mathrm{p}=0.258)$ on the latency of the fish to re-enter the light zone after the 395 first time visiting the dark zone in the light-dark test (Figure 7E).

Similar to the results found in the novel tank test in Experiment 1, caffeine-treated fish 397 generally appeared to be less exploratory, as caffeine-treated fish traveled less in the light zone,

398

399

400

401

402

403

404

405 406

made fewer crossings, and entered the light zone fewer times. Taurine generally did not alter overall exploration, and it does not appear that taurine has any mitigating or additive effects on caffeine-induced alterations in exploratory behavior in the light-dark test when both drugs were administered at the same time.

Whole-body cortisol levels post-light-dark test. A neurochemical marker of anxiety was determined by analyzing whole-body cortisol levels of each subject (Figure 8). A two-factor ANOVA revealed no significant main effect of caffeine $(F(1,35)=0.243, p=0.625)$, no significant main effect of taurine $(F(1,35)=0.274, p=0.604)$, and no significant interaction between caffeine and taurine $(\mathrm{F}(1,35)=0.024, \mathrm{p}=0.879)$ on whole body cortisol levels (Figure 
407 8). Thus, it does not appear that acute exposure to different components of energy drinks altered 408 stress hormone responses, at least when cortisol was sampled immediately after behavioral 409 measures were assessed in the light-dark test.

410

411

412

413

414

415

416

417

418

419

420

421

422

423

424

425

426

427

428

429

\section{Discussion}

The purpose of this study was to identify, in a zebrafish model, the various behavioral and neurochemical changes elicited in response to three treatments compared to control: caffeine, taurine, and caffeine and taurine in combination. Based on previous studies, it was expected that caffeine treatment would elicit anxiogenic effects, taurine treatment would elicit anxiolytic effects, and exposure to both caffeine and taurine would result in mixed effects on cortisol levels and behavioral measures associated with anxiety.

The results of this study indicate there are mixed effects of drug treatment on the various behavioral measures; however, in general, it appears that caffeine is anxiogenic in both the novel tank and light-dark tests. Taurine does not appear to have anxiolytic effects on its own, nor does it significantly impact the effects of caffeine when the two drugs are administered simultaneously, at least at the dose and time tested in the current study. In addition, there is no effect of drug treatment on whole-body cortisol levels in zebrafish.

The neurochemical analysis performed after each of the behavioral tests suggests that acute exposure to caffeine, taurine, or both does not affect basal levels of whole-body cortisol. Although previous studies have indicated that caffeine can alter basal levels of cortisol, the modulating effects of taurine on cortisol responses are only evident in response to acute stress (Mezzomo et al., 2019). Thus, the differences between the current and previously published studies are likely to be an outcome of methodological differences between experiments, such as 
430 varying durations of treatment and timing of behavioral measurements. Although behavioral

431 alterations persist in response to acute caffeine exposure (Tran et al., 2017), the time course of

432 the cortisol response may not necessarily parallel behavioral alterations induced by

433 pharmacological agents or stressors. In adult zebrafish, whole-body cortisol levels peak at 15

434 minutes in response to acute stressors (Ramsay et al., 2009; Tran, Chatterjee, \& Gerlai, 2014). In

435 the current study, cortisol was assessed 15 minutes after introduction to the behavioral task; thus,

436 it is possible that any perturbations in the cortisol levels elicited by the drug treatments may have

437 returned back to basal levels by the time of the assessment. One previous study assessed cortisol

438 levels immediately after recording behavioral measurements and demonstrated that a dose and

439 exposure time to caffeine comparable to the one used in the current study (100 mg for 15

440 minutes) significantly elevated whole-body cortisol levels compared to zebrafish not exposed to

441 caffeine (Rosa et al., 2018). Further studies should investigate whether caffeine, taurine, and a

442 mixture of these two drugs alter basal levels of cortisol immediately after drug exposure or if

443 these compounds alter peak cortisol responses after exposure to an acute stressor, such as a two-

444 minute net chase or exposure to conspecific alarm pheromone. The current results indicate that

445 acute exposure to the different treatments do not appear to elicit longer-term alterations (i.e. 30

446 minutes after the beginning of drug exposure) in basal levels of cortisol. Alternatively, these

447 treatments may not significantly affect basal cortisol at all, as has been observed with salivary

448 cortisol levels in human participants (Giles et al., 2012).

449 With regards to the behavioral assays, the only treatment to significantly impact behavior

450 was caffeine alone. Caffeine treatment significantly decreased the distance traveled, increased

451 resting time, and increased scototaxis (light avoidance) in the light-dark test and decreased

452 exploration of the top zone in the novel tank test. These findings support previous studies that 
453 indicate caffeine induces a heightened anxiety-like state (Egan et al., 2009; Richendrfer et al., 454 2012; Rosa et al., 2018; Schnörr et al., 2012; Steenbergen et al., 2011). Taurine treatment alone 455 did not appear to influence anxiety-like behavior, as measures on the different behavioral 456 parameters did not reach statistical significance. These findings are similar to the results from a 457 previously published study that demonstrated that taurine treatment alone had no measurable 458 effect on anxiety-like behavior in the novel tank test (Mezzomo et al., 2016). In that same study, 459 however, one hour of exposure to taurine did alter scototaxis in the light-dark task, as subjects 460 spent more time in the lit portion of the tank, suggesting an anxiolytic effect in this behavioral 461 test with longer exposure to treatment (Mezzomo et al., 2016). In the current study, a shorter 462 exposure time of fifteen minutes was used to keep the taurine treatment time equivalent to the 463 caffeine treatment. Thus, taurine alone may directly modulate anxiolytic behavior, but only in 464 certain behavioral paradigms with a minimum exposure time of greater than 15 minutes. To the 465 best of our knowledge, a full time course of the anxiolytic effects of taurine in either behavioral 466 task has yet to be investigated. Perhaps an even longer exposure time ( $>$ one hour) would be 467 sufficient to elicit behavioral effects in the novel tank test as well. A potential confound to this 468 study may be the feeding regimen of the zebrafish. Currently, there is no standardized diet or 469 feeding regimen across zebrafish colonies (Watts, Lawrence, Powell, \& D’Abramo, 2016). 470 However, a recent study suggests that feeding zebrafish once per day is associated with 471 decreased exploratory behavior compared to fish that were fed twice per day (Dametto et al., 472 2018). Although all of the fish in the current study were fed similarly, it is possible that any 473 potential effects of treatment may have been masked by anxiety induced by the feeding regimen. The current study is the first to examine the potential interaction of taurine and caffeine 475 on anxiety-like measures. At least at the dose and time course of taurine exposure used in the 
476 current study, it does not appear that taurine mitigates the anxiogenic effects of caffeine when

477 subjects are exposed to both drugs simultaneously. Further studies should investigate whether a

478 longer exposure time to taurine would mitigate the effects of caffeine on anxiety-like behavior,

479 and, if so, what neural mechanism would best explain these effects. As taurine exposure has been

480 shown to be anxiolytic in other studies in the literature, potentially, caffeine and taurine could

481 modulate anxiety-like behavior via similar neural targets. Some shared molecular targets include

482 adenosine and $\gamma$-amino butyric acid (GABA). Adenosine receptors are involved with modulating

483 anxiety in humans, rodents, and zebrafish (López-Cruz et al., 2017; Maximino et al., 2011;

484 Prediger, Batista, \& Takahashi, 2004; Prediger, da Silva, Batista, Bittencourt, \& Takahashi,

485 2006; Vincenzi, Borea, \& Varani, 2017; Yamada, Kobayashi, \& Kanda, 2014). Caffeine

486 antagonizes adenosine receptors (Ribeiro and Sebastião, 2010). Specifically, blockade of

487 adenosine $\mathrm{A}_{1}$ receptors in zebrafish attenuates the anxiogenic effect of caffeine (Maximino et al.,

488 2011). Higher doses or longer exposure to taurine could potentially elicit an increase in brain

489 levels of adenosine (Rosemberg et al., 2010), which may partially overcome the caffeine

490 antagonism of adenosine receptors. Alternatively, modulation of GABA transmission may be a

491 possible mechanism by which caffeine and taurine could regulate anxiety-like behavior. The

492 inhibitory activity of GABA is directly associated with anxiety-like responses (for review, see

493 Nuss, 2015). Both caffeine and taurine may be moderating the activity of GABAergic cells, as

494 administration of caffeine blocks GABAergic inhibitory postsynaptic potentials (IPSPs)

495 (Isokawa, 2016) and taurine enhances the activity of GABAergic interneurons (Sava, Chen, Sun,

496 Luhmann, \& Kilb, 2014). Although there is evidence to suggest that both caffeine and taurine

497 can mediate opposing functions within the same neurotransmitter system (e.g., adenosine or

498 GABA), and thus have the potential to modulate anxiety by a shared circuit, it is entirely possible 
499 that each of these compounds could be modulating separate systems to alter anxiety-like

500 behavior. Future studies should investigate whether caffeine and taurine are working on either

501 (or both) of these putative regulators of anxiety-like behavior, or if one or both of these

502 compounds modulate some other system entirely, such taurine modulating the glycine system

503 (Zhang \& Kim, 2007). Further studies should also investigate the impact of this acute regulation

504 of neural targets on downstream effects of the HPA/HPI system.

505

Future studies should also address the impact of caffeine, taurine, and mixed drug

506

exposure on different strains of zebrafish; some strains, such as the leopard strain, appear to have

507 higher baseline levels of anxiety (Egan et al., 2009). Future studies should also investigate

508 whether these compounds affect behavioral and neuroendocrine responses differently in male

509 and female fish. Stress-related behavior may vary in zebrafish depending on sex, as has been

510 observed in many other species (Donner \& Lowry, 2013). Sex differences in exploratory and

511 other behavioral responses in zebrafish have been studied less than other species (Ampatzis \&

512 Dermon, 2016), but indicate that sex may be a major factor in responsiveness to pharmacological

513 manipulations (Singer, Oreschak, Rhinehart, \& Robison, 2016).

514 Although the current study did not demonstrate that fifteen minutes of exposure to taurine

515 modulated caffeine-induced anxiety-like behavior, it is the first to study this question. More

516 studies are required to fully elucidate any synergistic effect of caffeine and taurine, as has been

517 observed in other measures, and whether activity of the HPA/HPI axis is involved with the

518 regulation of anxiety-like behavior. In addition, many more studies are needed to determine

519 whether anxiety-like states are altered by human consumption of highly caffeinated beverages

520 with significant taurine concentrations (e.g., energy drinks). It is also important to note that

521 energy drinks often contain many more additives that may or may not alter the properties of the 
522 two compounds under investigation of the current study. Also, given the previous literature,

523 whether taurine supplementation would be a viable avenue for treating anxiety conditions in

524 humans should be a focus of future investigations.

525

526

527

528

529

530

531

532

533

534

535

536

537

538

539

540

541

542

543 Dominguez, Alyssa Fassoth, Brianna Henning, Aleesa Parker, Summer Pattison, Adeel Shafiq,

544 Jessica Singh, Horace Townsend, Elijah Weathersby, and Jennifer Wright for their technical 
545 assistance with some aspects of this study. The use of Ethovision XT software (Noldus) was 546 made possible by the Faculty for Undergraduate Neuroscience (FUN) Equipment Loan program.

547

548

549

550

551

552

553

554

555

556

557

558

559

560

561

562

563

564

565

566

567

\section{References}

Attipoe, S., Delahanty, L., Stephens, M., \& Deuster, P. A. (2018). Energy Beverage Use Among U.S. Service Members. Military Medicine. https://doi.org/10.1093/milmed/usy169

Bichler, A., Swenson, A., \& Harris, M. A. (2006). A combination of caffeine and taurine has no effect on short term memory but induces changes in heart rate and mean arterial blood pressure. Amino Acids, 31(4), 471-476. https://doi.org/10.1007/s00726-005-0302-x

Cachat, J., Stewart, A., Grossman, L., Gaikwad, S., Kadri, F., Chung, K. M., ... Kalueff, A. V. (2010). Measuring behavioral and endocrine responses to novelty stress in adult zebrafish. Nature Protocols, 5(11), 1786-1799. https://doi.org/10.1038/nprot.2010.140

Canavello, P. R., Cachat, J. M., Beeson, E. C., Laffoon, A. L., Grimes, C., Haymore, W. A. M., ... Kalueff, A. V. (2011). Measuring Endocrine (Cortisol) Responses of Zebrafish to Stress. In Neuromethods: Vol. 51. Zebrafish Neurobehavioral Protocols (pp. 135-142).

Caviness, C. M., Anderson, B. J., \& Stein, M. D. (2017). Energy drinks and alcohol-related risk among young adults. Substance Abuse, 38(4), 376-381. 
569 Chaban, R., Kornberger, A., Branski, N., Buschmann, K., Stumpf, N., Beiras-Fernandez, A., \& Vahl, C. F. (2017). In-vitro examination of the positive inotropic effect of caffeine and

573 Chen, S. W., Kong, W. X., Zhang, Y. J., Li, Y. L., Mi, X. J., \& Mu, X. S. (2004). Possible anxiolytic effects of taurine in the mouse elevated plus-maze. Life Sciences, 75(12), 1503-1511. https://doi.org/10.1016/j.1fs.2004.03.010

576 Curran, C. P., \& Marczinski, C. A. (2017). Taurine, caffeine, and energy drinks: Reviewing the 577 risks to the adolescent brain. Birth Defects Research, 109(20), 1640-1648. https://doi.org/10.1002/bdr2.1177

Dametto, F. S., Fior, D., Idalencio, R., Rosa, J. G. S., Fagundes, M., Marqueze, A., ... Barcellos, 580 L. J. G. (2018). Feeding regimen modulates zebrafish behavior. PeerJ, 6, e5343. https://doi.org/10.7717/peerj.5343

Davis, D. J., Klug, J., Hankins, M., Doerr, H. M., Monticelli, S. R., Song, A., ... Bryda, E. C. (2015). Effects of Clove Oil as a Euthanasia Agent on Blood Collection Efficiency and Serum Cortisol Levels in Danio rerio. Journal of the American Association for

De Sanctis, V., Soliman, N., Soliman, A. T., Elsedfy, H., Di Maio, S., El Kholy, M., \& Fiscina, B. (2017). Caffeinated energy drink consumption among adolescents and potential health consequences associated with their use: a significant public health hazard. Acta BioMedica: Atenei Parmensis, 88(2), 222-231. https://doi.org/10.23750/abm.v88i2.6664

Dhanasiri, A. K. S., Fernandes, J. M. O., \& Kiron, V. (2013). Acclimation of zebrafish to 
transport stress. Zebrafish, 10(1), 87-98. https://doi.org/10.1089/zeb.2012.0843

592 Donner, N. C., \& Lowry, C. A. (2013). Sex differences in anxiety and emotional behavior. Pflugers Archiv: European Journal of Physiology, 465(5), 601-626.

594 https://doi.org/10.1007/s00424-013-1271-7

595

596

597

598

599

600

601

602

603

604

605

606

607

608

609

610

611

612

613

Egan, R. J., Bergner, C. L., Hart, P. C., Cachat, J. M., Canavello, P. R., Elegante, M. F., ... Kalueff, A. V. (2009). Understanding behavioral and physiological phenotypes of stress and anxiety in zebrafish. Behavioural Brain Research, 205(1), 38-44. https://doi.org/10.1016/j.bbr.2009.06.022

El Idrissi, A., Boukarrou, L., Heany, W., Malliaros, G., Sangdee, C., \& Neuwirth, L. (2009). Effects of taurine on anxiety-like and locomotor behavior of mice. Advances in Experimental Medicine and Biology, 643, 207-215.

Evans, J., \& Battisti, A. S. (2018). Caffeine. In StatPearls. Retrieved from http://www.ncbi.nlm.nih.gov/books/NBK519490/

Francisco, E. da S., \& Guedes, R. C. A. (2015). Neonatal taurine and alanine modulate anxietylike behavior and decelerate cortical spreading depression in rats previously suckled under different litter sizes. Amino Acids, 47(11), 2437-2445. https://doi.org/10.1007/s00726-015-2036-8

Giles, G. E., Mahoney, C. R., Brunyé, T. T., Gardony, A. L., Taylor, H. A., \& Kanarek, R. B. (2012). Differential cognitive effects of energy drink ingredients: caffeine, taurine, and glucose. Pharmacology, Biochemistry, and Behavior, 102(4), 569-577. https://doi.org/10.1016/j.pbb.2012.07.004

Harper, C., \& Lawrence, C. (2016). The Laboratory Zebrafish. Boca Raton: CRC Press. Heckman, M. A., Weil, J., \& Gonzalez de Mejia, E. (2010). Caffeine (1, 3, 7-trimethylxanthine) 
614

615

616

617

618

619

620

621

622

623

624

625

626

627

628

629

630

631

632

633

634

635

636

in foods: a comprehensive review on consumption, functionality, safety, and regulatory matters. Journal of Food Science, 75(3), R77-87. https://doi.org/10.1111/j.17503841.2010.01561.x

Higgins, J. P., Babu, K., Deuster, P. A., \& Shearer, J. (2018). Energy Drinks: A Contemporary Issues Paper. Current Sports Medicine Reports, 17(2), 65-72. https://doi.org/10.1249/JSR.0000000000000454

Isokawa, M. (2016). Caffeine-Induced Suppression of GABAergic Inhibition and CalciumIndependent Metaplasticity. Neural Plasticity, 2016, 1239629. https://doi.org/10.1155/2016/1239629

Kimura, M., Ushijima, I., Hiraki, M., Kimura, M., \& Ono, N. (2009). Enhancement of caffeineinduced locomotor hyperactivity produced by the combination with L-arginine or taurine in mice: Possible involvement of nitric oxide. Methods and Findings in Experimental and Clinical Pharmacology, 31(9), 585-589. https://doi.org/10.1358/mf.2009.31.9.1435462

Kong, W. X., Chen, S. W., Li, Y. L., Zhang, Y. J., Wang, R., Min, L., \& Mi, X. (2006). Effects of taurine on rat behaviors in three anxiety models. Pharmacology, Biochemistry, and Behavior, 83(2), 271-276. https://doi.org/10.1016/j.pbb.2006.02.007

Kysil, E. V., Meshalkina, D. A., Frick, E. E., Echevarria, D. J., Rosemberg, D. B., Maximino, C., ... Kalueff, A. V. (2017). Comparative Analyses of Zebrafish Anxiety-Like Behavior Using Conflict-Based Novelty Tests. Zebrafish, 14(3), 197-208. https://doi.org/10.1089/zeb.2016.1415

López-Cruz, L., Carbó-Gas, M., Pardo, M., Bayarri, P., Valverde, O., Ledent, C., ... Correa, M. (2017). Adenosine A2A receptor deletion affects social behaviors and anxiety in mice: Involvement of anterior cingulate cortex and amygdala. Behavioural Brain Research, 
637

638

639

640

641

642

643

644

645

646

647

648

649

650

651

652

653

654

655

656

657

658

659

321, 8-17. https://doi.org/10.1016/j.bbr.2016.12.020

Magno, L. D. P., Fontes, A., Gonçalves, B. M. N., \& Gouveia, A. (2015). Pharmacological study of the light/dark preference test in zebrafish (Danio rerio): Waterborne administration. Pharmacology, Biochemistry, and Behavior, 135, 169-176. https://doi.org/10.1016/j.pbb.2015.05.014

Manchester, J., Eshel, I., \& Marion, D. W. (2017). The Benefits and Risks of Energy Drinks in Young Adults and Military Service Members. Military Medicine, 182(7), e1726-e1733. https://doi.org/10.7205/MILMED-D-16-00339

Maximino, C., da Silva, A. W. B., Araújo, J., Lima, M. G., Miranda, V., Puty, B., ... Herculano, A. M. (2014). Fingerprinting of psychoactive drugs in zebrafish anxiety-like behaviors. PloS One, 9(7), e103943. https://doi.org/10.1371/journal.pone.0103943

Maximino, C., Lima, M. G., Olivera, K. R. M., Picanço-Diniz, D. L. W., \& Herculano, A. M. (2011). Adenosine A1, but not A2, receptor blockade increases anxiety and arousal in Zebrafish. Basic \& Clinical Pharmacology \& Toxicology, 109(3), 203-207. https://doi.org/10.1111/j.1742-7843.2011.00710.x

Maximino, C., Marques de Brito, T., Dias, C. A. G. de M., Gouveia, A., \& Morato, S. (2010). Scototaxis as anxiety-like behavior in fish. Nature Protocols, 5(2), 209-216. https://doi.org/10.1038/nprot.2009.225

McCool, B. A., \& Chappell, A. (2007). Strychnine and taurine modulation of amygdalaassociated anxiety-like behavior is "state" dependent. Behavioural Brain Research, 178(1), 70-81. https://doi.org/10.1016/j.bbr.2006.12.002

Mezzomo, N. J., Fontana, B. D., Müller, T. E., Duarte, T., Quadros, V. A., Canzian, J., ... Barcellos, L. J. G. (2019). Taurine modulates the stress response in zebrafish. Hormones 
660

and Behavior, 109, 44-52. https://doi.org/10.1016/j.yhbeh.2019.02.006

661 Mezzomo, N. J., Silveira, A., Giuliani, G. S., Quadros, V. A., \& Rosemberg, D. B. (2016). The

662 role of taurine on anxiety-like behaviors in zebrafish: A comparative study using the

663 novel tank and the light-dark tasks. Neuroscience Letters, 613, 19-24.

664 https://doi.org/10.1016/j.neulet.2015.12.037

665 National Research Council. (2011). Guide for the Care and Use of Laboratory Animals (8th ed.).

666 Washington DC: The National Academies Press.

667 Nehlig, A. (2018). Interindividual Differences in Caffeine Metabolism and Factors Driving

668 Caffeine Consumption. Pharmacological Reviews, 70(2), 384-411.

669 https://doi.org/10.1124/pr.117.014407

670 Nesan, D., \& Vijayan, M. M. (2013). Role of glucocorticoid in developmental programming:

671 evidence from zebrafish. General and Comparative Endocrinology, 181, 35-44.

672 https://doi.org/10.1016/j.ygcen.2012.10.006

673 Oja, S. S., \& Saransaari, P. (2017). Significance of Taurine in the Brain. Advances in

674 Experimental Medicine and Biology, 975 Pt 1, 89-94. https://doi.org/10.1007/978-94-

675 024-1079-2_8

676 Owoyele, B. V., Oyewole, A. L., Biliaminu, S. A., \& Alashi, Y. (2015). Effect of taurine and

677 caffeine on plasma c-reactive protein and calcium in Wistar rats. African Journal of

678 Medicine and Medical Sciences, 44(3), 229-236.

679 Peacock, A., Martin, F. H., \& Carr, A. (2013). Energy drink ingredients. Contribution of caffeine

680 and taurine to performance outcomes. Appetite, 64, 1-4.

$681 \quad$ https://doi.org/10.1016/j.appet.2012.12.021

682 Prediger, R. D. S., Batista, L. C., \& Takahashi, R. N. (2004). Adenosine A1 receptors modulate 
683

684

685

686

687

688

689

690

691

692

693

694

695

696

697

698

699

700

701

702

703

704

705

the anxiolytic-like effect of ethanol in the elevated plus-maze in mice. European Journal of Pharmacology, 499(1-2), 147-154. https://doi.org/10.1016/j.ejphar.2004.07.106

Prediger, R. D. S., da Silva, G. E., Batista, L. C., Bittencourt, A. L., \& Takahashi, R. N. (2006). Activation of adenosine A1 receptors reduces anxiety-like behavior during acute ethanol withdrawal (hangover) in mice. Neuropsychopharmacology: Official Publication of the American College of Neuropsychopharmacology, 31(10), 2210-2220. https://doi.org/10.1038/sj.npp.1301001

Raymond, J., Chanin, S., Stewart, A. M., Kyzar, E., Gaikwad, S., Roth, A., ... Kalueff, A. V. (2012). Assessing Habituation Phenotypes in Adult Zebrafish: Intra-and Inter-Trial Habituation in the Novel Tank Test. In Neuromethods: Vol. 66. Zebrafish Protocols for Neurobehavioral Research (pp. 273-285). Totowa NJ: Humana Press.

Richards, G., \& Smith, A. P. (2016). A Review of Energy Drinks and Mental Health, with a Focus on Stress, Anxiety, and Depression. Journal of Caffeine Research, 6(2), 49-63. https://doi.org/10.1089/jcr.2015.0033

Richendrfer, H., Pelkowski, S. D., Colwill, R. M., \& Creton, R. (2012). On the edge: pharmacological evidence for anxiety-related behavior in zebrafish larvae. Behavioural Brain Research, 228(1), 99-106. https://doi.org/10.1016/j.bbr.2011.11.041

Ripps, H., \& Shen, W. (2012). Review: taurine: a "very essential" amino acid. Molecular Vision, $18,2673-2686$.

Rosa, L. V., Ardais, A. P., Costa, F. V., Fontana, B. D., Quadros, V. A., Porciúncula, L. O., \& Rosemberg, D. B. (2018). Different effects of caffeine on behavioral neurophenotypes of two zebrafish populations. Pharmacology, Biochemistry, and Behavior, 165, 1-8. https://doi.org/10.1016/j.pbb.2017.12.002 
706 Sava, B. A., Chen, R., Sun, H., Luhmann, H. J., \& Kilb, W. (2014). Taurine activates

707

708

709

710

711

712

713

714

715

716

717

718

719

720

721

722

723

724

725

726

727

728

GABAergic networks in the neocortex of immature mice. Frontiers in Cellular Neuroscience, 8, 26. https://doi.org/10.3389/fncel.2014.00026

Schaffer, S. W., Shimada, K., Jong, C. J., Ito, T., Azuma, J., \& Takahashi, K. (2014). Effect of taurine and potential interactions with caffeine on cardiovascular function. Amino Acids, 46(5), 1147-1157. https://doi.org/10.1007/s00726-014-1708-0

Schnörr, S. J., Steenbergen, P. J., Richardson, M. K., \& Champagne, D. L. (2012). Measuring thigmotaxis in larval zebrafish. Behavioural Brain Research, 228(2), 367-374. https://doi.org/10.1016/j.bbr.2011.12.016

Seidl, R., Peyrl, A., Nicham, R., \& Hauser, E. (2000). A taurine and caffeine-containing drink stimulates cognitive performance and well-being. Amino Acids, 19(3-4), 635-642.

Steenbergen, P. J., Richardson, M. K., \& Champagne, D. L. (2011). Patterns of avoidance behaviours in the light/dark preference test in young juvenile zebrafish: a pharmacological study. Behavioural Brain Research, 222(1), 15-25. https://doi.org/10.1016/j.bbr.2011.03.025

Stewart, A., Gaikwad, S., Kyzar, E., Green, J., Roth, A., \& Kalueff, A. V. (2012). Modeling anxiety using adult zebrafish: a conceptual review. Neuropharmacology, 62(1), 135-143. https://doi.org/10.1016/j.neuropharm.2011.07.037

Stewart, A., Maximino, C., de Brito, T. M., Herculano, A. M., Gouveia, A., Morato, S., ... Kalueff, A. V. (2011). Neurophenotyping of Adult Zebrafish Using the Light/Dark Box Paradigm. In Neuromethods: Vol. 51. Zebrafish Neurobehavioral Protocols. Springer Science+Business Media, LLC.

Tallis, J., Higgins, M. F., Cox, V. M., Duncan, M. J., \& James, R. S. (2014). Does a 
729

730

731

732

733

734

735

736

737

738

739

740

741

742

743

744

745

746

747

748

749

750

751

physiological concentration of taurine increase acute muscle power output, time to fatigue, and recovery in isolated mouse soleus (slow) muscle with or without the presence of caffeine? Canadian Journal of Physiology and Pharmacology, 92(1), 42-49. https://doi.org/10.1139/cjpp-2013-0195

Trunzo, J. J., Samter, W., Morse, C., McClure, K., Kohn, C., Volkman, J. E., \& O’Brien, K. (2014). College students' use of energy drinks, social problem-solving, and academic performance. Journal of Psychoactive Drugs, 46(5), 396-401. https://doi.org/10.1080/02791072.2014.965291

Valle, M. T. C., Couto-Pereira, N. S., Lampert, C., Arcego, D. M., Toniazzo, A. P., Limberger, R. P., ... Leal, M. B. (2018). Energy drinks and their component modulate attention, memory, and antioxidant defences in rats. European Journal of Nutrition, 57(7), 25012511. https://doi.org/10.1007/s00394-017-1522-z

Vercammen, K. A., Koma, J. W., \& Bleich, S. N. (2019). Trends in Energy Drink Consumption Among U.S. Adolescents and Adults, 2003-2016. American Journal of Preventive Medicine, 56(6), 827-833. https://doi.org/10.1016/j.amepre.2018.12.007

Vincenzi, F., Borea, P. A., \& Varani, K. (2017). Anxiolytic properties of A1 adenosine receptor PAMs. Oncotarget, 8(5), 7216-7217. https://doi.org/10.18632/oncotarget.13802

Warnock, R., Jeffries, O., Patterson, S., \& Waldron, M. (2017). The Effects of Caffeine, Taurine, or Caffeine-Taurine Coingestion on Repeat-Sprint Cycling Performance and Physiological Responses. International Journal of Sports Physiology and Performance, 12(10), 1341-1347. https://doi.org/10.1123/ijspp.2016-0570

Watts, S. A., Lawrence, C., Powell, M., \& D’Abramo, L. R. (2016). The Vital Relationship Between Nutrition and Health in Zebrafish. Zebrafish, 13 Suppl 1, S72-76. 
753 Wendelaar Bonga, S. E. (1997). The stress response in fish. Physiological Reviews, 77(3), 591754 625. https://doi.org/10.1152/physrev.1997.77.3.591

755 Westerfield, M. (2000). The zebrafish book: A guide for the laboratory use of zebrafish (Danio 756 rerio) (4th ed.). Eugene: University of Oregon Press.

757 Wong, D., von Keyserlingk, M. A. G., Richards, J. G., \& Weary, D. M. (2014). Conditioned 758 place avoidance of zebrafish (Danio rerio) to three chemicals used for euthanasia and anaesthesia. PloS One, 9(2), e88030. https://doi.org/10.1371/journal.pone.0088030

760

761

762

763

764

765

766

767

768

769

770

771

772

773

774

Wong, K., Elegante, M., Bartels, B., Elkhayat, S., Tien, D., Roy, S., ... Kalueff, A. V. (2010). Analyzing habituation responses to novelty in zebrafish (Danio rerio). Behavioural Brain Research, 208(2), 450-457. https://doi.org/10.1016/j.bbr.2009.12.023

Wu, G.-F., Ren, S., Tang, R.-Y., Xu, C., Zhou, J.-Q., Lin, S.-M., ... Yang, J.-C. (2017). Antidepressant effect of taurine in chronic unpredictable mild stress-induced depressive rats. Scientific Reports, 7(1), 4989. https://doi.org/10.1038/s41598-017-05051-3

Yamada, K., Kobayashi, M., \& Kanda, T. (2014). Involvement of adenosine A2A receptors in depression and anxiety. International Review of Neurobiology, 119, 373-393. https://doi.org/10.1016/B978-0-12-801022-8.00015-5

Zhang, C. G., \& Kim, S.-J. (2007). Taurine induces anti-anxiety by activating strychninesensitive glycine receptor in vivo. Annals of Nutrition \& Metabolism, 51(4), 379-386. https://doi.org/10.1159/000107687 


\section{Table 1 (on next page)}

Percentage of zebrafish that did not explore top zone in the novel tank test

Acute exposure to energy drink components (caffeine, taurine, or both (CAF+TAU)) did significantly influence the number of fish that failed to explore the top zone in the novel tank test in adult zebrafish. 
1 Table 1: Percentage of zebrafish that did not explore top zone

2

\begin{tabular}{ccccccc}
\hline Variable & Control & Caffeine & Taurine & CAF+TAU & & \\
& $(\mathrm{N}=41)$ & $(\mathrm{N}=19)$ & $(\mathrm{N}=19)$ & $(\mathrm{N}=20)$ & $\chi^{2}$ & \\
Did not explore top & $9.8 \%$ & $42.1 \%$ & $5.3 \%$ & $25.0 \%$ & 12.02 & 0.007 \\
\hline
\end{tabular}

3

4

5

6

7

8

9

10

11

12

13

14

15

16

17

18

19

20

21 


\section{Figure 1}

Measures of zebrafish motor activity in the novel tank test

Acute exposure to energy drink components (caffeine, taurine, or both) did not alter $(A)$ the total distance traveled and $(B)$ the mean speed while ambulatory in the novel tank test in adult zebrafish. Bars indicate means of each group \pm SEM. 

A

\section{Total Distance Traveled \\ Novel Tank Test}

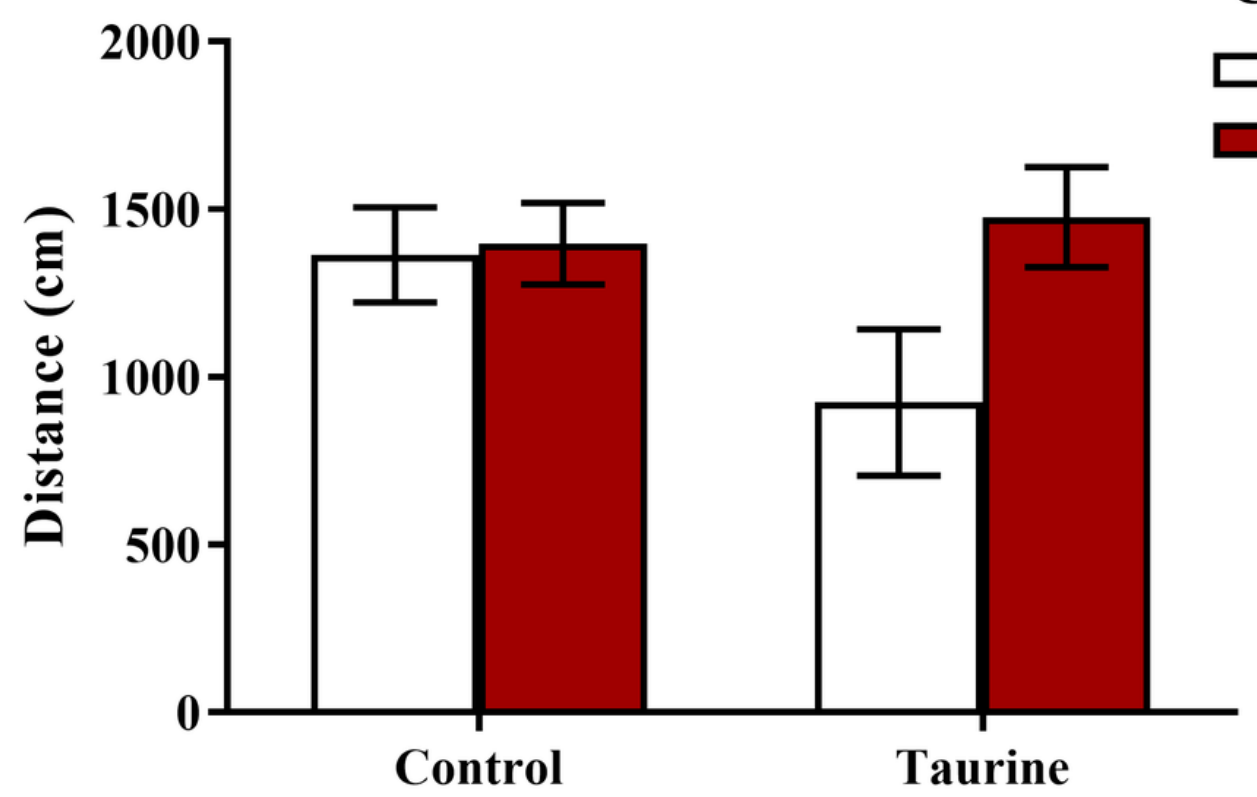

B

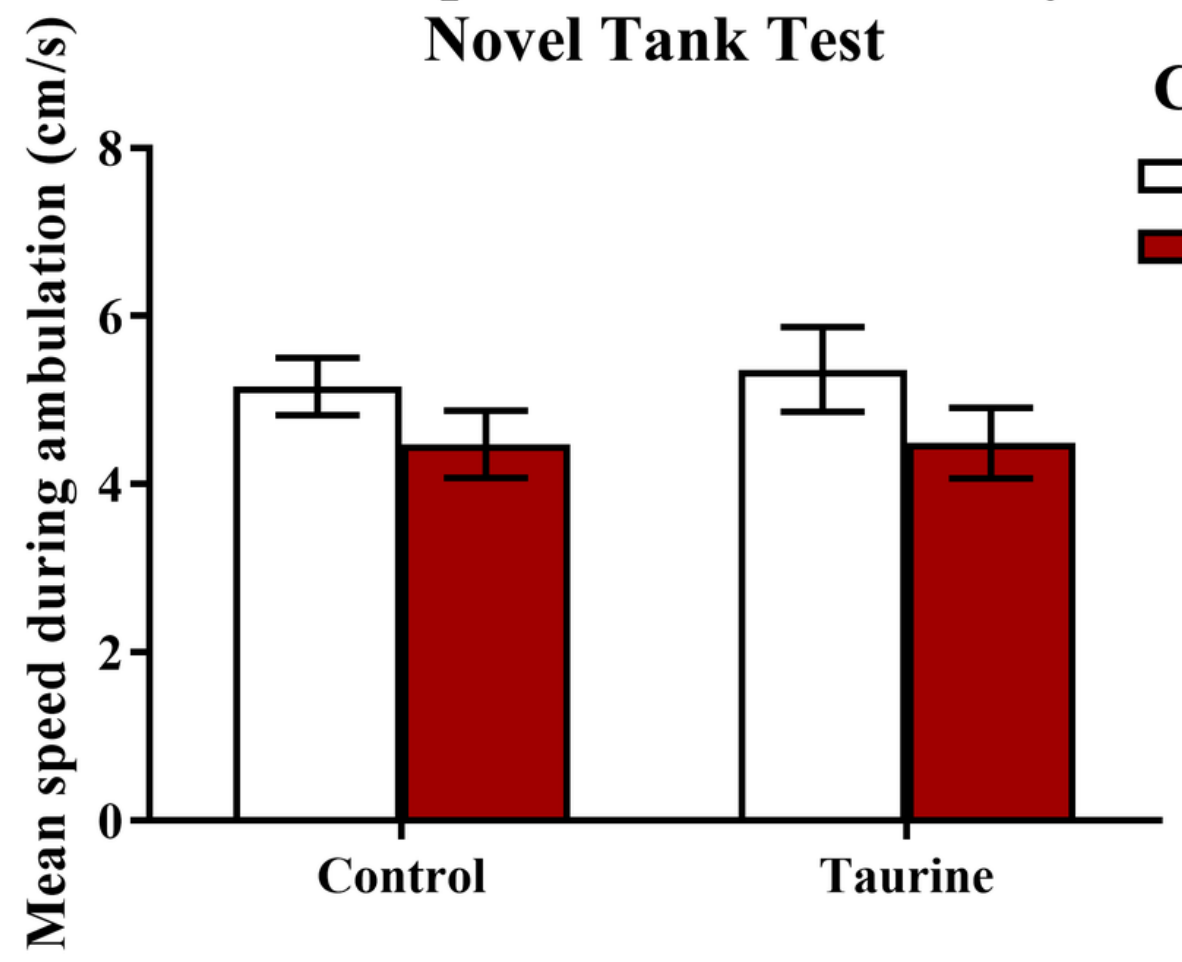

Caffeine

No

Yes
Caffeine

$\square$ No

$\square$ Yes 
Figure 2

Measures of zebrafish freezing behavior in the novel tank test

Acute exposure to energy drink components (caffeine, taurine, or both) did not alter $(A)$ the total number of immobility bouts and $(B)$ the total time spent immobile in the novel tank test in adult zebrafish. Bars indicate means of each group \pm SEM. 
A

Bouts of Immobility

Novel Tank Test

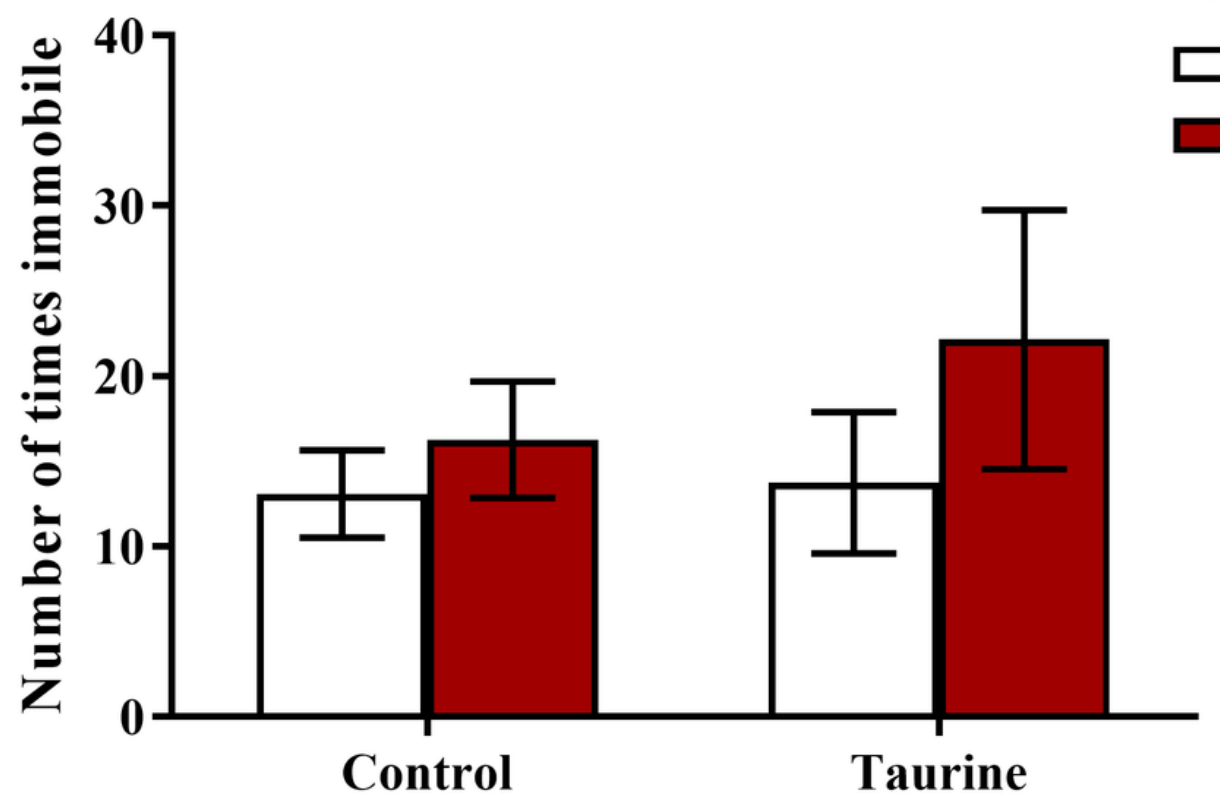

Caffeine

$\square$ No

$\square$ Yes

B

Time Immobile

Novel Tank Test

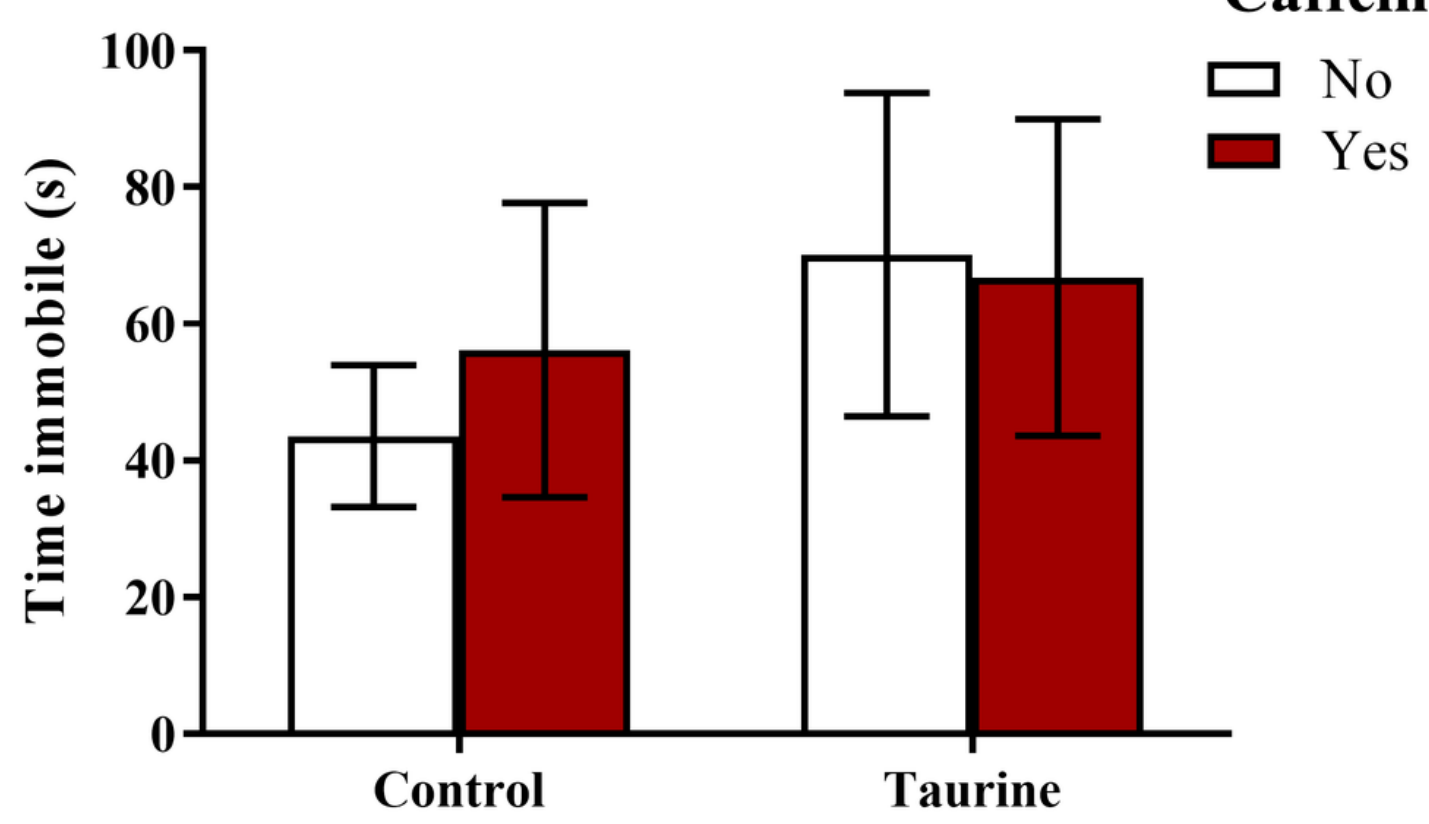




\section{Figure 3}

Measures of zebrafish exploratory behavior in the novel tank test

Acute exposure to energy drink components (caffeine, taurine, or both) altered exploratory behavior in the novel tank test in adult zebrafish. Caffeine decreased (A) the distance traveled in the top zone, (B) the number of entries to the top zone, and (C) the time spent in the top zone. Taurine decreased (D) the latency to enter the top zone. Bars indicate means of each group \pm SEM.

$\mathbf{A}$

Distance in Top

Novel Tank Test

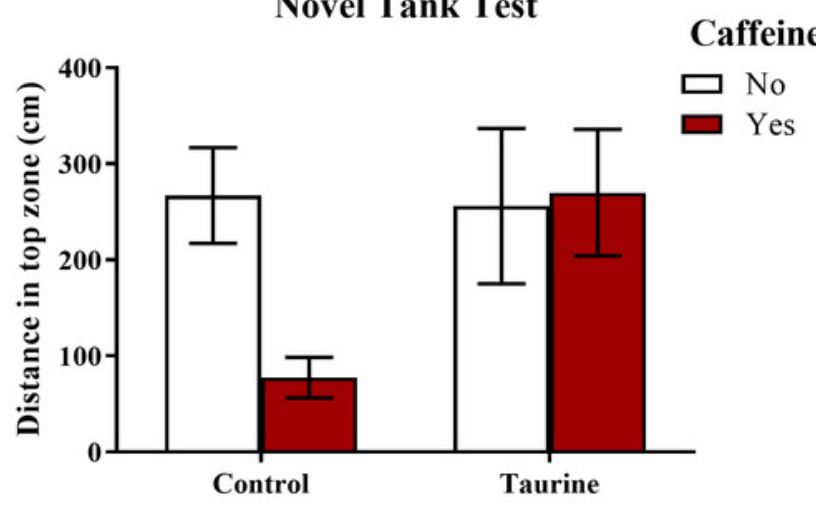

C

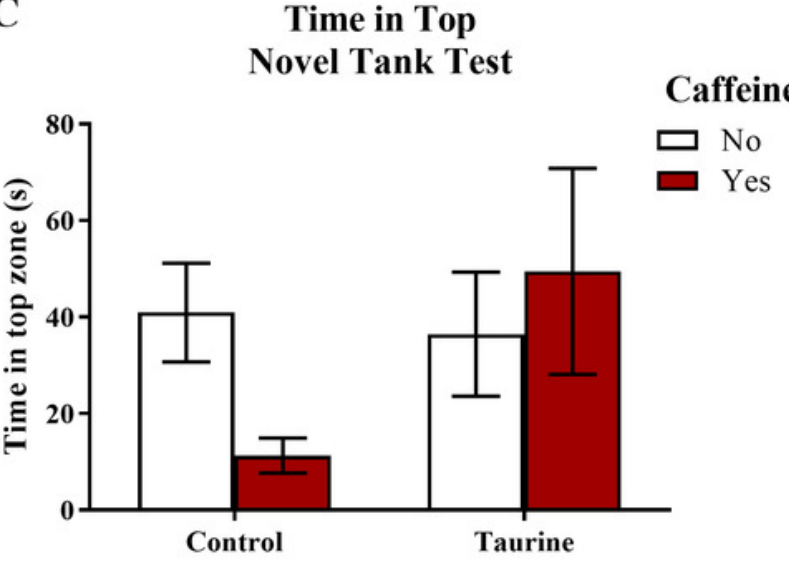

B

Entries to Top

Novel Tank Test

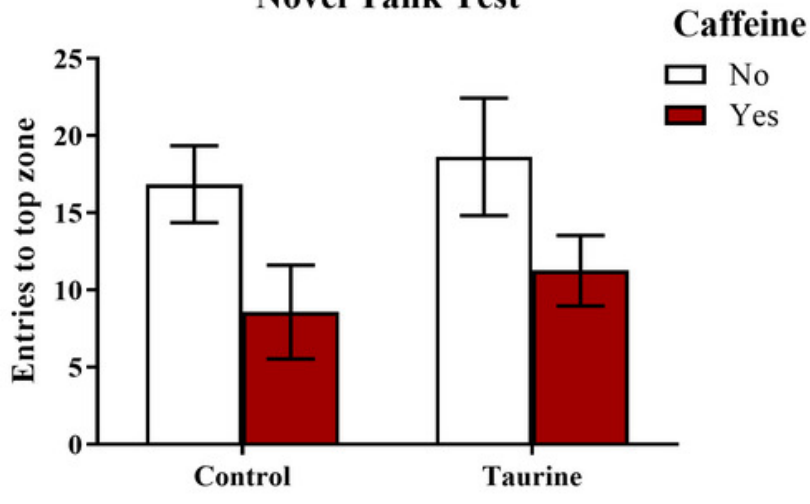

D Latency to Top

Novel Tank Test

Caffeine

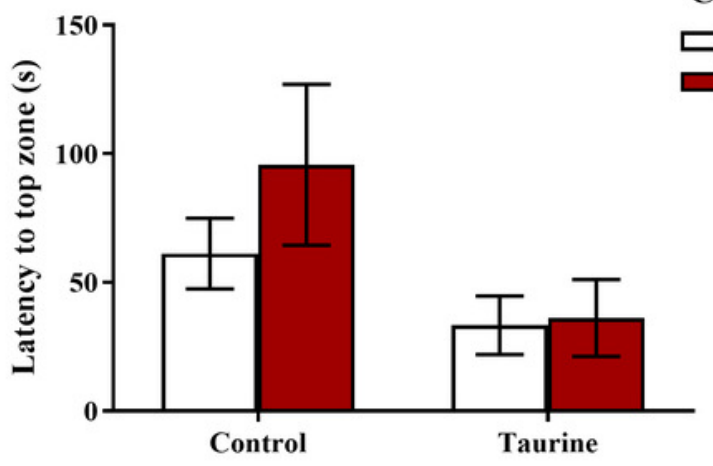


Figure 4

Measures of zebrafish neuroendocrine function after the novel tank test

Acute exposure to energy drink components (caffeine, taurine, or both) did not alter wholebody cortisol levels of zebrafish of fish in Experiment 1 (fish were sacrificed after the novel tank test). Bars indicate means of each group \pm SEM.

\section{Cortisol \\ (After Novel Tank Test)}

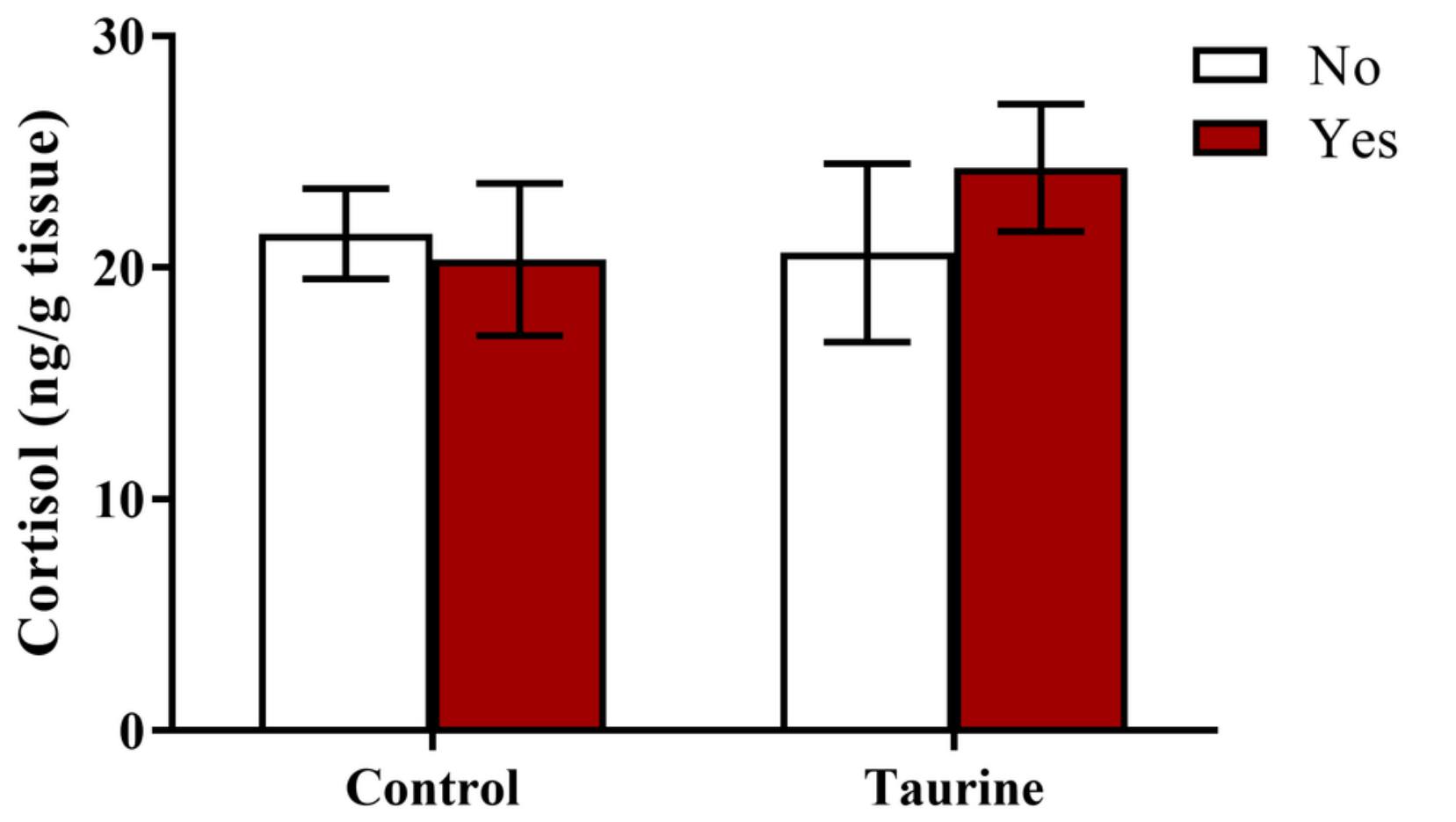




\section{Figure 5}

Measures of zebrafish motor activity in the light-dark test

Acute exposure to energy drink components (caffeine, taurine, or both) affected some aspects of motor activity in adult zebrafish. Acute caffeine decreased (A) the total distance traveled but not (B) the mean speed during ambulation in the light-dark test. Bars indicate means of each group \pm SEM and $* *$ indicates significant $(p<0.01)$ difference from respective non-caffeine treated control (Tukey post-hoc). 

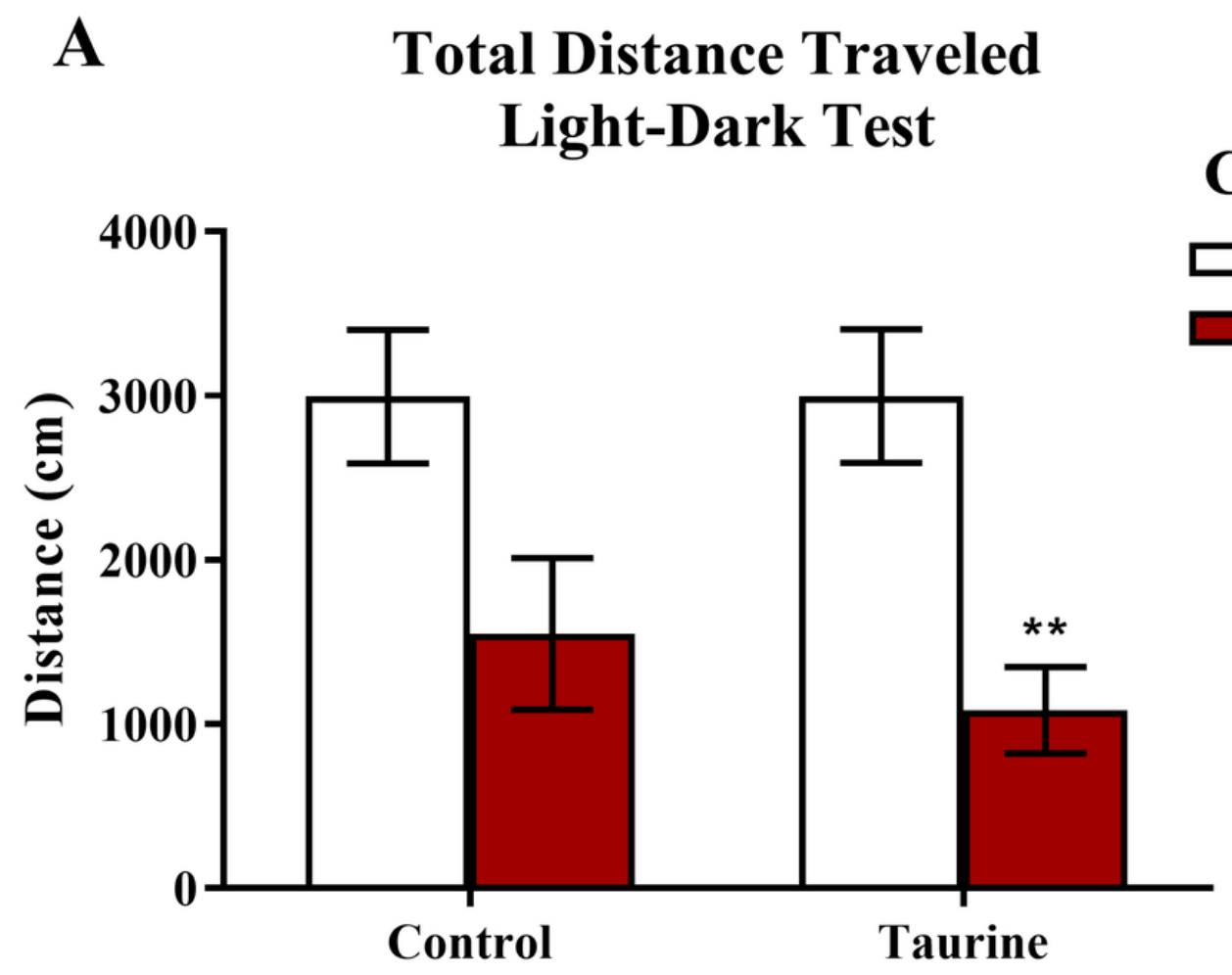

B

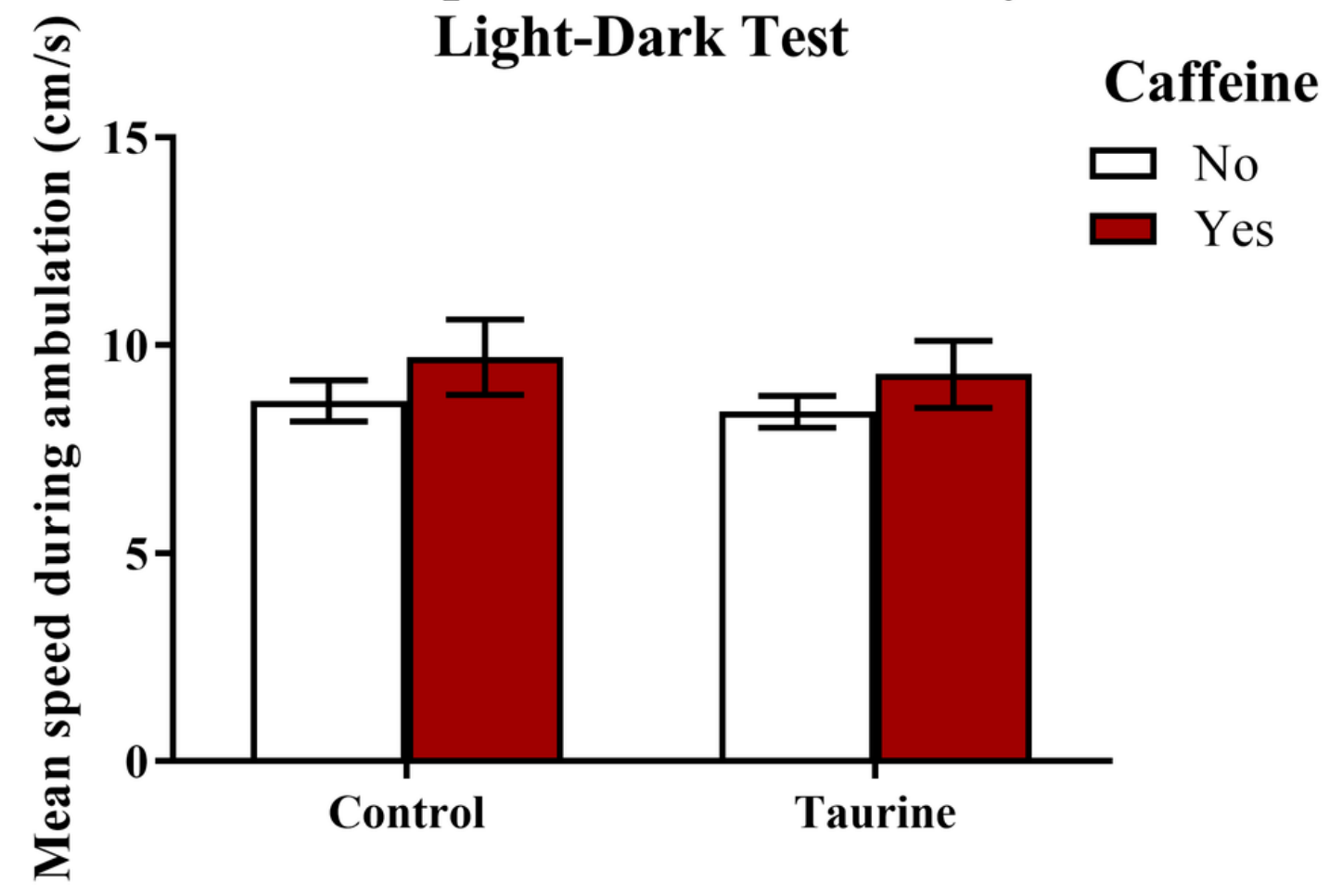

B Mean Speed while Ambulatory
Caffeine

No

$\square$ Yes 


\section{Figure 6}

Measures of zebrafish freezing behavior in the light-dark test

Acute exposure to energy drink components (caffeine, taurine, or both) altered the total time spent immobile in the novel tank test in adult zebrafish. Caffeine increased the total time immobile in the light-dark test. Bars indicate means of each group \pm SEM. *,** indicates significant ( $p<0.05$ and $p<0.01$, respectively) difference from respective non-caffeine treated control (Tukey post-hoc).

\section{Time Immobile Light-Dark Test}

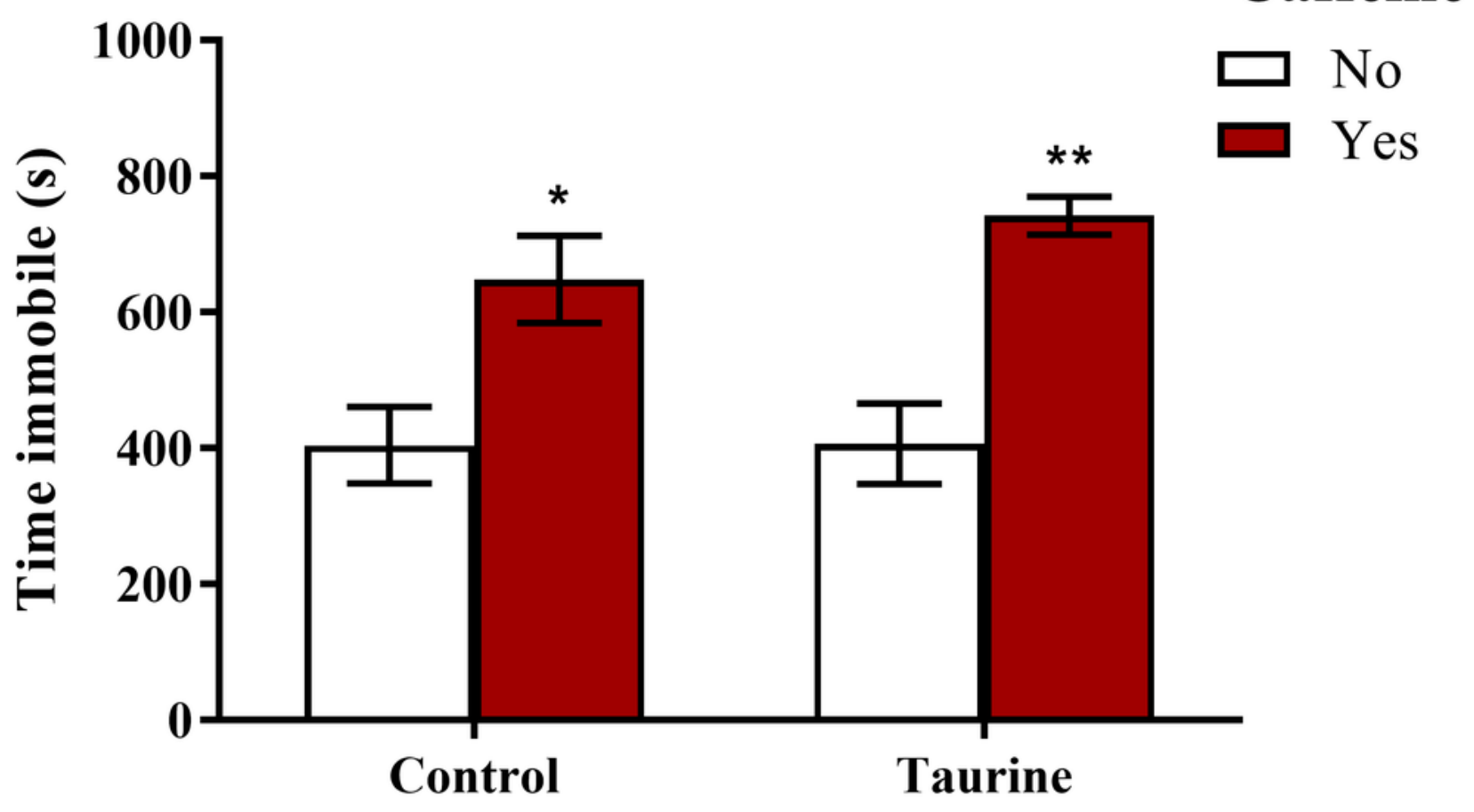




\section{Figure 7}

Measures of zebrafish exploratory behavior in the light-dark test

Acute exposure to energy drink components (caffeine, taurine, or both) altered exploratory behavior in the novel tank test in adult zebrafish. Caffeine decreased (A) the distance traveled in the light zone, (B) the number of entries to the light zone, and $(C)$ the number of crossings between compartments. The acute drug treatments did not significantly alter (D) the time spent in the light zone nor $(\mathrm{E})$ the latency to re-enter the light zone after the first visit to the dark zone. Bars indicate means of each group \pm SEM. *,** indicates significant ( $p$ $<0.05$ and $p<0.01$, respectively) difference from respective non-caffeine treated control (Tukey post-hoc).
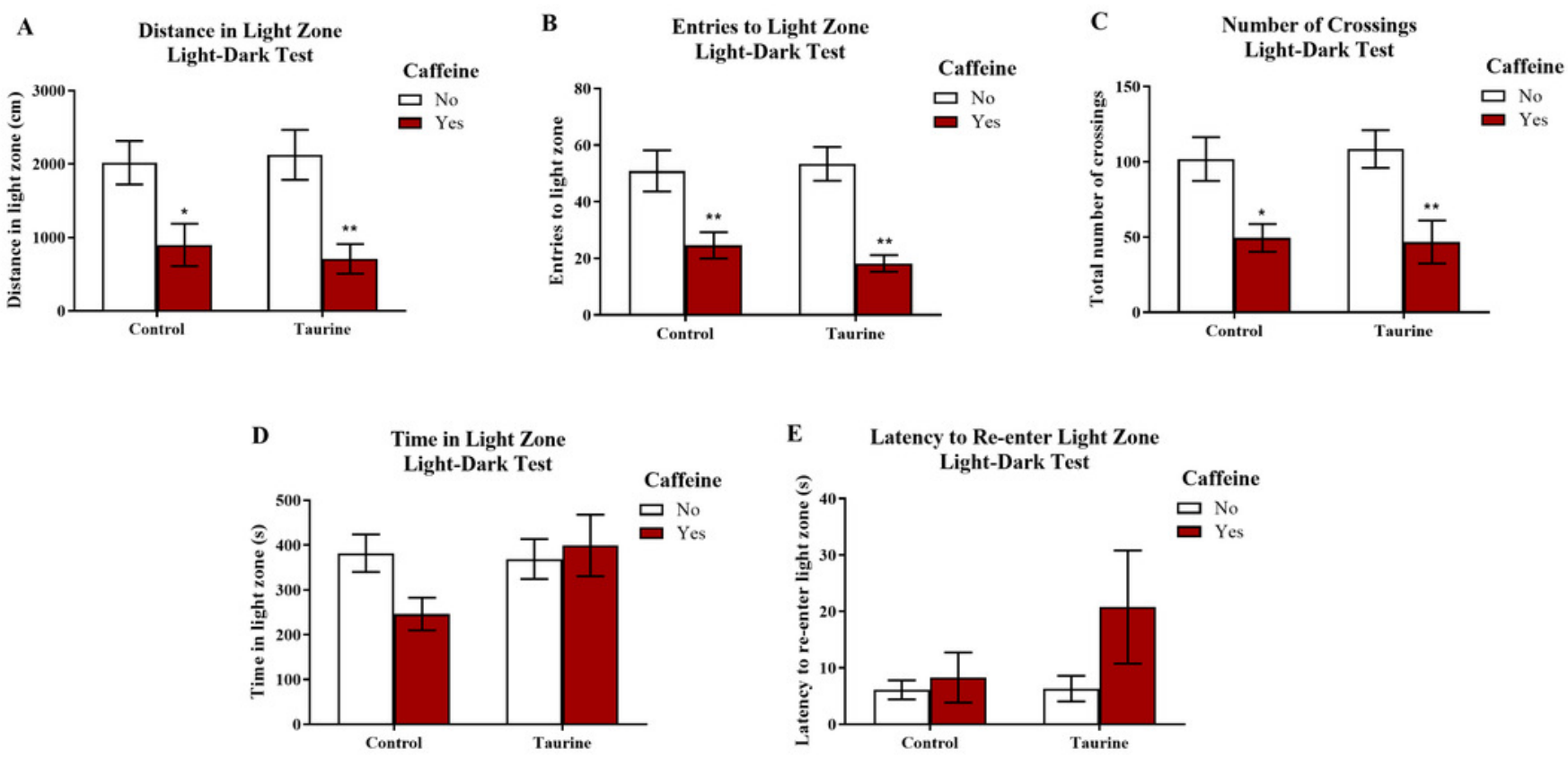


\section{Figure 8}

Measures of zebrafish neuroendocrine function after the light-dark test

Acute exposure to energy drink components (caffeine, taurine, or both) did not alter wholebody cortisol levels of zebrafish of fish in Experiment 2 (fish were sacrificed after the lightdark test). Bars indicate means of each group \pm SEM.

\section{Cortisol \\ (After Light-Dark Test)}

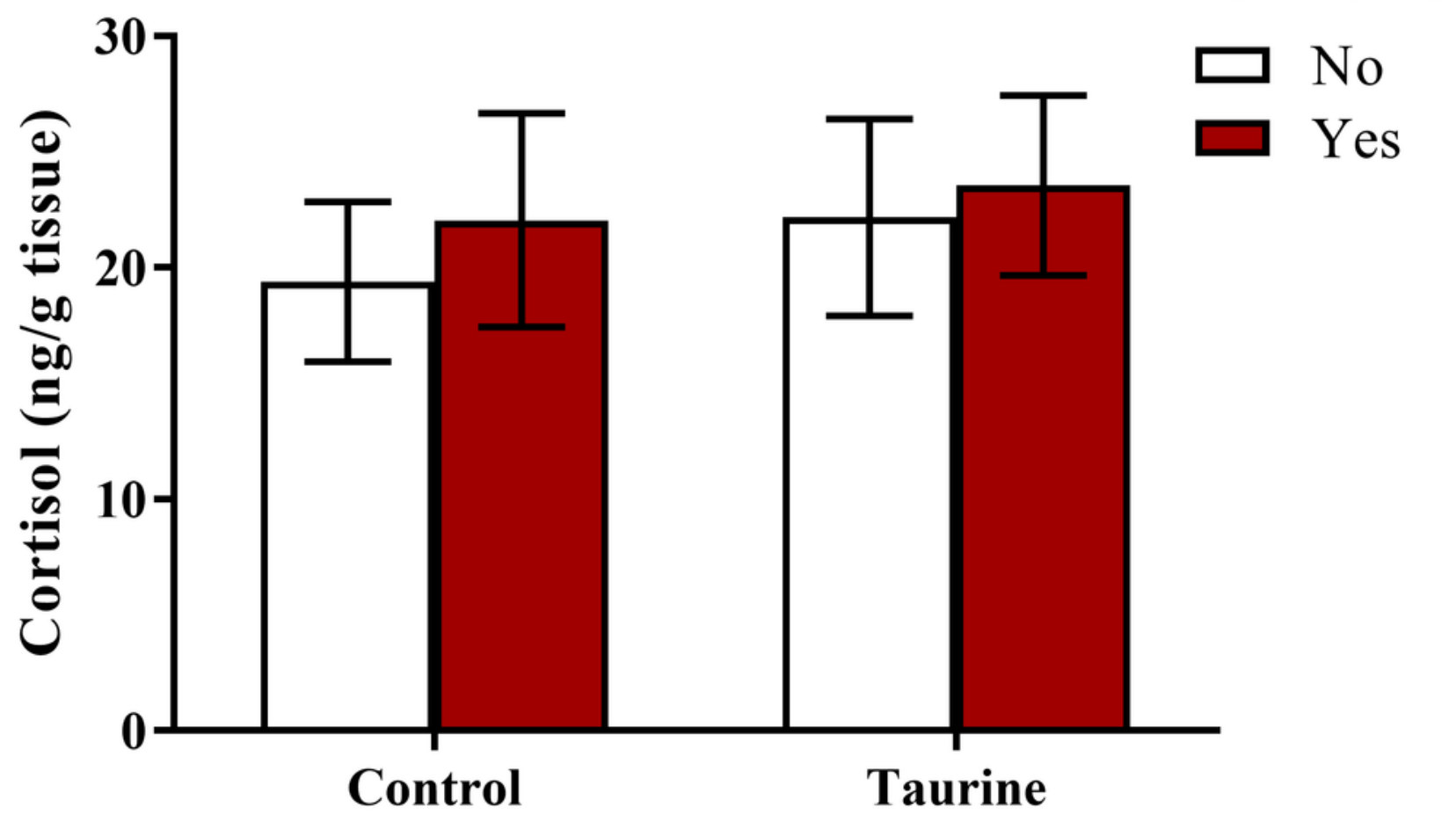

